\title{
Activation of Ezrin/Radixin/Moesin Mediates Attractive Growth Cone Guidance through Regulation of Growth Cone Actin and Adhesion Receptors
}

\author{
Bonnie M. Marsick, Jose E. San Miguel-Ruiz, and Paul C. Letourneau \\ Department of Neuroscience, Graduate Program in Neuroscience, University of Minnesota, Minneapolis, Minnesota 55455
}

\begin{abstract}
The development of a functioning neural network relies on responses of axonal growth cones to molecular guidance cues that are encountered en route to their target tissue. Nerve growth factor (NGF) and neurotrophin-3 serve as attractive cues for chick embryo sensory growth cones in vitro and in vivo, but little is known about the actin-binding proteins necessary to mediate this response. The evolutionarily conserved ezrin/radixin/moesin (ERM) family of proteins can tether actin filaments to the cell membrane when phosphorylated at a conserved threonine residue. Here we show that acute neurotrophin stimulation rapidly increases active phospho-ERM levels in chick sensory neuron growth cone filopodia, coincident with an increase in filopodial L1 and $\beta$-integrin. Disrupting ERM function with a dominant-negative construct (DN-ERM) results in smaller and less motile growth cones with disorganized actin filaments. Previously, we found that NGF treatment increases actin-depolymerizing factor (ADF)/cofilin activity and growth cone F-actin (Marsick et al., 2010). Here, we show this F-actin increase, as well as attractive turning to NGF, is blocked when ERM function is disrupted despite normal activation of ADF/cofilin. We further show that DN-ERM expression disrupts leading edge localization of active ADF/cofilin and free F-actin barbed ends. Moreover, filopodial phospho-ERM levels are increased by incorporation of active ADF/cofilin and reduced by knockdown of L1CAM.Together, these data suggest that ERM proteins organize actin filaments in sensory neuron growth cones and are crucial for neurotrophin-induced remodeling of F-actin and redistribution of adhesion receptors.
\end{abstract}

\section{Introduction}

Proper synaptic development depends on pathfinding by axonal growth cones (Lowery and Van Vactor, 2009; Kolodkin and Tessier-Lavigne, 2011). Growth cones detect extrinsic cues and respond by actin filament reorganization that drives growth cone motility (Huber et al., 2003; Gallo and Letourneau, 2004; Geraldo and Gordon-Weeks, 2009; Lowery and Van Vactor, 2009). Turning toward attractants involves leading edge protrusion toward the attractant (Lin and Forscher, 1993; Kalil and Dent, 2005). Previously, we reported that the attractant nerve growth factor (NGF) induces actin polymerization in growth cone regions closer to the attractant (Marsick et al., 2010). Activation of the actin-severing protein $\mathrm{ADF} /$ cofilin (where $\mathrm{ADF}$ is actindepolymerizing factor) mediates this stimulated polymerization; yet it is unknown how these new actin filaments interact with the membrane to drive protrusion.

\footnotetext{
Received Sept. 19, 2011; revised 0ct. 31, 2011; accepted Nov. 4, 2011

Author contributions: B.M.M., J.E.S.M.-R., and P.C.L. designed research; B.M.M. and J.E.S.M.-R. performed research; B.M.M., J.E.S.M.-R., and P.C.L. analyzed data; B.M.M. and P.C.L. wrote the paper.

Funding support was provided by National Eye Institute Training Grant EY07133 (B.M.M.) and NIH Grant HD 19950. We thank Florence Roche for technical assistance, Dr. James R. Bamburg and Alisa Shaw (Colorado State University) for assistance in recombinant XACA3 protein purification and for generously providing the CA-LIMK plasmid, and Dr. Murray Blackmore for design of the L1 morpholinos.

The authors declare no competing financial interests.

Correspondence should be addressed to Paul C Letourneau, Department of Neuroscience, Graduate Program in Neuroscience, 6-145 Jackson Hall, University of Minnesota, Minneapolis, MN 55455. E-mail: letou001@umn.edu. DOI:10.1523/JNEUROSCI.4794-11.2012

Copyright $\odot 2012$ the authors $\quad 0270-6474 / 12 / 320282-15 \$ 15.00 / 0$
}

Few actin-regulatory proteins have well characterized roles in axon guidance (Pak et al., 2008; Lowery and Van Vactor, 2009). The conserved ezrin/radixin/moesin (ERM) proteins often organize membrane-cytoskeletal interactions (Amieva and Furthmayr, 1995; Tsukita and Yonemura, 1999; Bretscher et al., 2002; Niggli and Rossy, 2008). ERMs share a C-terminal actin-binding domain and an N-terminal FERM domain that binds membrane proteins such as CD44, ICAM1, L1 cell adhesion molecule, and dystrophin (Bretscher et al., 2002; McClatchey and Fehon, 2009). ERMs also act as scaffolding for adaptor and signaling molecules. ERM activity is conformationally regulated. Phosphorylation of a conserved threonine residue disrupts an $\mathrm{N}-\mathrm{C}$ termini interaction to allow ERMs to link F-actin and membranes (Tsukita et al., 1997; Simons et al., 1998; Hayashi et al., 1999). Thus, ERM proteins are key components in organizing membranes, cytoskeletal-membrane interactions, and cell signaling.

ERM proteins are expressed in growth cones and may mediate actin-membrane interactions (Amieva and Furthmayr, 1995; Gonzalez-Agosti and Solomon, 1996; Paglini et al., 1998; Castelo and Jay, 1999; Niggli and Rossy, 2008) ERMs bind L1, an important axonal adhesion receptor (Dickson et al., 2002; Mintz et al., 2003; Cheng et al., 2005; Tang et al., 2007; Sakurai et al., 2008). Growth cone repulsion by Semaphorin 3A may involve ERM inactivation (Gallo, 2008; Mintz et al., 2008), and studies showing ERM activation downstream of NGF in PC12 cells make ERMs good candidates for mediating growth cone responses to NGF (Jeon et al., 2010). Thus, ERMs could facilitate axon guidance by regulating F-actin organization and the distribution of membrane proteins. 
We report here that gradients of attractive cues NGF and neurotrophin-3 (NT3) locally activate ERM proteins in growth cones of chick sensory neurons. Activated ERMs colocalize at the leading edge with L1, ADF/cofilin, and F-actin barbed ends. When ERM protein levels are reduced or ERM function is disrupted by a dominant-negative construct (DN-ERM), neurite length and growth cone F-actin levels are reduced. Moreover, DN-ERM blocks NGF-induced increases in F-actin and filopodial adhesion receptors, as well as turning toward NGF. Directly increasing ADF/cofilin activity increases phospho-ERM levels, and knockdown of the ERM binding partner L1, conversely, reduces filopodial phospho-ERM. These results provide insights into the role of ERM proteins in organizing actin filaments and adhesion receptors to promote leading edge protrusion and chemoattractive responses.

\section{Materials and Methods}

Materials. F-12 medium, B27 additives, laminin, poly-D-lysine (molecular weight, >300,000), Alexa Fluor 488 DNase1, Alexa Fluor 488- and 568-phalloidin, and Alexa Fluor 488 and 568 secondary antibodies were purchased from Invitrogen. NGF, NT3, L1, and N-cadherin were purchased from R \& D Systems. LY-294002 was purchased from Cell Signaling Technology. Chariot was purchased from Active Motif. White Leghorn fertilized chicken eggs were purchased from Hy-Line. All other drugs and reagents were purchased from Sigma-Aldrich unless otherwise indicated.

Neuronal culture. Glass coverslips (Gold Seal) were coated with 100 $\mu \mathrm{g} / \mathrm{ml}$ poly-D-lysine, rinsed three times with water, dried, coated with $5 \%$ nitrocellulose dissolved in $100 \%$ amyl acetate (Fischer Scientific), dried, and coated overnight with $20 \mu \mathrm{g} / \mathrm{ml}$ laminin or $4 \mu \mathrm{g} / \mathrm{ml} \mathrm{L1}$ in PBS (Roche). Video dishes were made by gluing (silicone aquarium sealant) a coverslip ( $18 \times 18 \mathrm{~mm}$; Gold Seal) over a hole $(5 \mathrm{~mm})$ in the bottom of a culture dish $(35 \times 10 \mathrm{~mm}$; Falcon), allowed to dry, rinsed with water, and coated as described. Embryonic day 7 dorsal root ganglia (DRGs) were removed from chick embryos of either sex according to procedures approved by the University of Minnesota Institutional Animal Care and Use Committee (Minneapolis, MN). Neural tissues were cultured on experimental substrates in F-12 with added B27, glutamine, sodium pyruvate, and glucose and buffered to $\mathrm{pH} 7.4$ with $10 \mathrm{mM}$ HEPES. For low calcium media, a 1:1 solution was made of F-12 and calcium-free Hank's balanced salt solution with added B27, glutamine, sodium pyruvate, and glucose. For the high calcium condition, calcium chloride was added to $1.0 \mathrm{~mm}$ to this medium. Neural tissues were cultured overnight or for $48 \mathrm{~h}$ in a humidified incubator at $37^{\circ} \mathrm{C}$.

Neuronal transfection. DRGs were dissociated as described by Roche et al. (2009). Approximately $2 \times 10^{6}$ cells were transfected with one of the following: $1 \mu \mathrm{g}$ of plasmid expressing GFP, $1 \mu \mathrm{g}$ of a plasmid encoding GFP tagged to the $\mathrm{N}$ terminus of ezrin (DN-ERM; a generous gift from Dr. Janis Burkhardt, University of Pennsylvania, Philadelphia PA), 0.5 $\mu \mathrm{g}$ of GFP combined with siRNA against chick radixin (10 nM) and moesin (10 nM), or $2 \mu \mathrm{g}$ of constitutively active LIMK (CA-LIMK; a generous gift from Dr. James R. Bamburg, Colorado State University, Fort Collins, CO) mixed with $1 \mu \mathrm{g}$ of GFP plasmid using the G-13 program of the Lonza Nucleofector and the chicken neuron Nucleofector reagents. To assess retrograde actin flow or for barbed end or active ADF labeling studies, $2 \mu \mathrm{g}$ of GFP or DN-ERM were combined with $0.5 \mu \mathrm{g}$ of plasmids for RFP- or GFP-actin (a kind gift from James R. Bamburg, Colorado State University, Fort Collins, CO). Morpholinos against chick L1 and control morpholinos were purchased from Gene Tools; sequence and transfection protocol were described by Blackmore and Letourneau (2006b). Short interfering RNA (siRNA) oligonucleotides were purchased from Invitrogen and sequences were as follows: chick radixin antisense, 5'-ACGUUGAUUGGUUUCGGCA-3'; chick moesin antisense, 5'-ACGCUGAUCGUUUUCGGCA-3'; scrambled control, 5' UCCUGGAUCUUAGUGCGUU-3'. Transfected cells were cultured 24 or $48 \mathrm{~h}$, as indicated.
Immunocytochemistry. Neuronal cultures were fixed and blocked as described by Roche et al. (2009). Coverslips were incubated with primary antibodies diluted in PBS containing $10 \%$ goat serum overnight at $4^{\circ} \mathrm{C}$. Affinity-purified antibody 12977 (ADF) was used at 1:200 dilution, and antibody 4321 (phospho-ADF/cofilin) was used at 1:1000 (generous gifts of Dr. James R. Bamburg, Colorado State University, Fort Collins, CO); ERM antibodies against ezrin, moesin, phospho-ERM, and pan-ERM (Cell Signaling Technology) were used at 1:100; 13H9 (pan-ERM; a generous gift from Lorene Lanier, University of Minnesota, Minneapolis, $\mathrm{MN}$ ) was used at 1:1000; antibody specific for radixin (R3653; SigmaAldrich) was used at 1:100; polyclonal antibody specific for radixin (457-3; a generous gift from Frank Solomon, Massachusetts Institute of Technology, Cambridge, MA) was used at 1:200; anti- $\beta 1$ integrin (W1B10; Sigma-Aldrich) was used at 1:100; monoclonal anti-chick-L1 (8D9; Developmental Studies Hybridoma Bank, University of Iowa, Iowa City, IA) was used at $10 \mu \mathrm{g} / \mathrm{ml}$; anti-N-cadherin (ab12221; Abcam) was used at 1:100; polyclonal anti-chick-TrkA (a kind gift from Dr. Frances Lefcort, Montana State University, Bozeman, MT); and anti-PAkt (Cell Signaling Technology) was used at 1:50. Coverslips were then rinsed three times in PBS and incubated in PBS rinse for $1 \mathrm{~h}$. For labeling F-actin, Alexa Fluor 568-phalloidin was applied at a 1:20 dilution and mixed with secondary antibodies Alexa Fluor 568 goat anti-rabbit or anti-mouse at 1:1000 dilution in PBS with 10\% goat serum for $1 \mathrm{~h}$. For labeling G-actin, $5 \mu \mathrm{g} / \mathrm{ml}$ Alexa Fluor 488 DNase1 in PBS was applied to coverslips for $1 \mathrm{~h}$. For staining total active ADF, medium was removed and cultures were incubated $1 \mathrm{~min}$ in permeabilization buffer (described below, Barbed end labeling) with $100 \mathrm{~nm}$ phalloidin and then fixed and stained. After rinsing three times in PBS, coverslips were mounted in SlowFade reagent (Invitrogen).

Time-lapse microscopy and quantitative fluorescence determinations. A Spot digital camera mounted on an Olympus XC-70 inverted microscope, and MetaMorph software (Molecular Devices) were used for all image acquisitions. In any one experiment, all images were acquired in one session. For repeat experiments, data were normalized to controls. For collection of fluorescent images, exposure time and gain settings on the digital camera were kept constant, and image acquisition and analysis was performed as described by Roche et al. (2009).

For experiments measuring growth cone fluorescence intensity values, a line tool in MetaMorph was used to outline the terminal $25 \mu \mathrm{m}$ of each distal axon and growth cone, and background intensity value was subtracted from the fluorescence intensity value of the accompanying neuronal measurement. For phospho-ERM and L1 filopodial intensity measurements, growth cones were selected at random from phalloidin images, and a line was drawn on each filopodium using the line tool; lines were then transferred to the corresponding phospho-ERM or L1 image, and background-subtracted intensity values were recorded. To measure the intensity of $\beta$-integrin at filopodial tips, phalloidin images were used to draw a $20 \times 20$ pixel box at the tip of each filopodium. Box regions were then transferred to the corresponding integrin image, and background-subtracted intensity values were recorded.

Central and peripheral region quantifications of active $\mathrm{ADF} / \mathrm{cofilin}$ and barbed labeling were performed by hand tracing GFP-actin images in MetaMorph. A straight line was then drawn across the growth cone width in at least three locations, and the distance $25 \%$ from either side of the growth cone edge was noted and used to trace a central growth cone region. Regions were then transferred to the $\mathrm{ADF} /$ cofilin or rhodamineactin image, where total and central growth cone intensities were measured. Peripheral growth cone intensities were later calculated by subtracting the central intensity from the total for each growth cone.

Proximal/distal filopodial intensity measurements in gradients were performed as follows. Growth cones were subjected to a gradient of BSA, NGF, NT3, chariot, or chariot + A3 and were imaged, fixed, and process for immunochemistry. In MetaMorph, a line along the neurite axis was extended forward to divide the growth cone in two. A line was drawn on each filopodium toward the cue source (proximal) and away from the cue source (distal), and the background-subtracted intensity values were measured. For each growth cone, the filopodia intensity values were averaged for each half and used to calculate the proximal/distal ratio, as described by Marsick et al. (2010). 
Retrograde flow measurements. To determine retrograde actin flow rates, DRGs were cotransfected to express RFP-actin and either GFP or DN-ERM and cultured overnight with NGF. Cotransfected growth cones were identified, and RFP-actin was imaged every $3 \mathrm{~s}$ for $6 \mathrm{~min}$. Kymographs were generated for the distal $10 \mu \mathrm{m}$ growth cones, and flow rates were measured by tracking bright RFP-actin features, which are formed by unequal incorporation of RFP- and non-RFP-actin monomers into polymerized filaments at the leading edge. Measuring retrograde flow using the movement of these features has been described previously (Marsick et al., 2010), including a demonstrated sensitivity of this retrograde flow to the myosin-II inhibitor blebbistatin (Chan and Odde, 2008).

Pharmacological inhibitors. Trk inhibitor K252a (500 nM), PI3K inhibitor LY-294002 (10 $\mu \mathrm{M})$, PLC inhibitor U73122 (1 $\mu \mathrm{M})$, or PKC inhibitor chelerythrine $(1 \mu \mathrm{M})$ were added to cultures for $15 \mathrm{~min}$ before media or NGF addition. Controls were treated with the same volume of DMSO vehicle, which never exceeded $5 \mu \mathrm{l} / \mathrm{ml}$.

Barbed end labeling. This protocol was adapted from Chan et al. (1998) and is described by Marsick et al. (2010). For colabeling with active ADF, cells were permeabilized and processed for barbed end labeling, fixed, and then stained for total ADF (12977). For colabeling with phosphoERM, $0.1 \%$ glutaraldehyde and $0.1 \%$ paraformaldehyde were added to permeabilization buffer. Following Rh-actin labeling, cells were further fixed $30 \mathrm{~min}$ and stained for phospho-ERM.

Recombinant proteins and protein loading. Recombinant XAC proteins were generated as described previously (Gehler et al., 2004). Proteins were delivered into cells using Chariot reagent (Active Motif; Morris et al., 2001) according to the manufacture's instructions. Briefly, $6 \mu \mathrm{l}$ of Chariot was complexed with $1 \mu \mathrm{g}$ of XACA3 for $1 \mathrm{~h}$ and then added to the culture medium or immobilized onto a nitrocellulose-coated micropipette.

Growth cone turning assay. Turning was assessed as described previously (Roche et al., 2009; Marsick et al., 2010). Briefly, micropipette tips were dipped in a $1 \%$ nitrocellulose solution, dried, and then dipped several times in a PBS solution of $1 \mu \mathrm{g} / \mathrm{ml}$ NGF or $1 \mathrm{ng} / \mathrm{ml} \mathrm{NT3}$. A growth cone was imaged at 30 or $60 \mathrm{~s}$ intervals for $15 \mathrm{~min}$. The micropipette tip was then positioned $100 \mu \mathrm{m}$ (NGF) or $50 \mu \mathrm{m}$ (NT3) from a growth cone at a $45^{\circ}$ angle to the direction of axon elongation. Images were acquired for $60 \mathrm{~min}$ after introducing the micropipette tip. Growth cone turning angles were determined as the change in direction of growth cone migration between the beginning and end of the image acquisition period (Ming et al., 1997).

\section{Results}

\section{Growth cone ERM proteins are activated by NGF}

Similar to previous reports, we found that ERM proteins are expressed in chick embryo DRG growth cones. Antibodies that recognize all three ERM proteins (pan-ERM, 13H9) stained growth cones strongly and co-distributed in the peripheral domain (P-domain) with phalloidin-labeled F-actin (Fig. 1A). Using antibodies specific to individual ERM proteins, we found strong radixin staining in the P-domain similar to pan-ERM staining, whereas staining for ezrin and moesin was strongest in the growth cone C-domain and neurite shaft (Fig. 1A). These immunostaining data suggest that radixin is the predominant ERM protein present in the DRG growth cone leading margin and peripheral domain, consistent with previous reports (Castelo and Jay, 1999).

ERM activation can be immunologically assessed with an antibody specific to threonine-phosphorylated ERM proteins in their active conformation (phospho-ERM or p-ERM). Using this antibody, we found that acute addition of $40 \mathrm{ng} / \mathrm{ml} \mathrm{NGF}$ increases levels of active ERM proteins, most predominantly in growth cone lamellipodia and filopodia (Fig. $1 B$ ). Experiments with timed NGF exposures demonstrate that active ERM levels increase rapidly following NGF addition and are then reduced and restored upon NGF removal and re-addition, respectively (Fig. 1B).
To assess ERM distribution in a model of axon guidance, a diffusible gradient of NGF was generated by placing an NGFcoated pipette tip near a growth cone for 5 min (Marsick et al., 2010), and then cells were fixed and stained for phospho-ERM. This resulted in elevated phospho-ERM in the growth cone regions closer to the NGF source, while no such asymmetry occurred in growth cones subjected to a gradient of BSA (Fig. 1C). These results indicate that acute NGF treatment increases active ERM levels in both a local and a global manner.

We next investigated the signaling that mediates NGFinduced ERM phosphorylation in DRG growth cones by using inhibitors of trkA activation and several downstream effectors. Previous studies have identified numerous kinases upstream of ERM phosphorylation, including molecules activated by neurotrophins or other growth factors (Gautreau et al., 1999; Matsui et al., 1999; Bretscher et al., 2002; Baumgartner et al., 2006; Gallo, 2008; Jeon et al., 2010; Antoine-Bertrand et al., 2011). DRG cultures were treated for 15 min with $500 \mathrm{~nm} \mathrm{k252a}$ (trkA), $10 \mu \mathrm{M}$ LY294002 (PI3 kinase), $1 \mu \mathrm{M}$ U73122 (PLC), or $1 \mu \mathrm{M}$ chelerythrine (PKC) before the global addition of control medium or 40 $\mathrm{ng} / \mathrm{ml} \mathrm{NGF}$ for $10 \mathrm{~min}$. The trk inhibitor k252a completely blocked ERM phosphorylation in response to NGF addition, while inhibiting PI3K, PLC, or PKC individually before NGF treatment lowered baseline phospho-ERM levels and resulted in lower phospho-ERM levels after NGF addition, compared to DMSO + NGF (Fig. 1D). However, only the trkA inhibitor completely blocked the NGF-induced increase in phospho-ERM, and the other inhibitors significantly but partially reduced the size of the NGF-induced increase in ERM phosphorylation (Fig. 1D). In agreement with previous studies, these data suggest that multiple signaling pathways can simultaneously regulate phospho-ERM levels (Yonemura et al., 2002; Jeon et al., 2010; Antoine-Bertrand et al., 2011).

\section{Neurotrophin-3 activates ERM proteins and induces attractive turning}

We next investigated whether ERM activity is regulated by another attractive guidance cue. Neurotrophin-3 attracts developing proprioceptive and trigeminal axons to their target tissues (O'Connor and Tessier-Lavigne, 1999; Genç et al., 2004) and facilitates the regeneration of adult sensory neurons (Ramer et al., 2000; 2002; Alto et al., 2009). Similar to NGF, we found that treatment of DRG explants for $15 \mathrm{~min}$ with $50 \mathrm{ng} / \mathrm{ml} \mathrm{NT3}$ increased filopodial phospho-ERM levels (Fig. $2 \mathrm{~A}$ ). Exposure to a localized source of NT3 diffusing from a micropipette increased proximal phospho-ERM levels (Fig. $2 B$ ) and induced attractive growth cone turning (Fig. 2C). Acute NT3 treatment also increased the levels of growth cone F-actin and active (dephosphorylated) $\mathrm{ADF} /$ cofilin (Fig. $2 \mathrm{~A}$ ). These data suggest that local activation of $\mathrm{ADF} /$ cofilin and ERM proteins mediate attractive growth cone turning to multiple guidance cues.

\section{NGF treatment colocalizes phospho-ERM, ADF/cofilin, and F-actin barbed ends at the leading edge and increases filopodial L1 and $\beta$-1integrin expression}

We previously reported that activation of ADF/cofilin mediates NGF-stimulated actin polymerization and growth cone turning toward an NGF source (Marsick et al., 2010). Here, we visualized the distribution of activated ERM in relation to $\mathrm{ADF} /$ cofilin and F-actin free barbed ends. As shown in Figure 3A, phospho-ERM co-distributes with active ADF/cofilin and rhodamine-labeled F-actin barbed ends at the leading edge of NGF-treated growth cones. Thus, active ERM proteins are appropriately localized so 


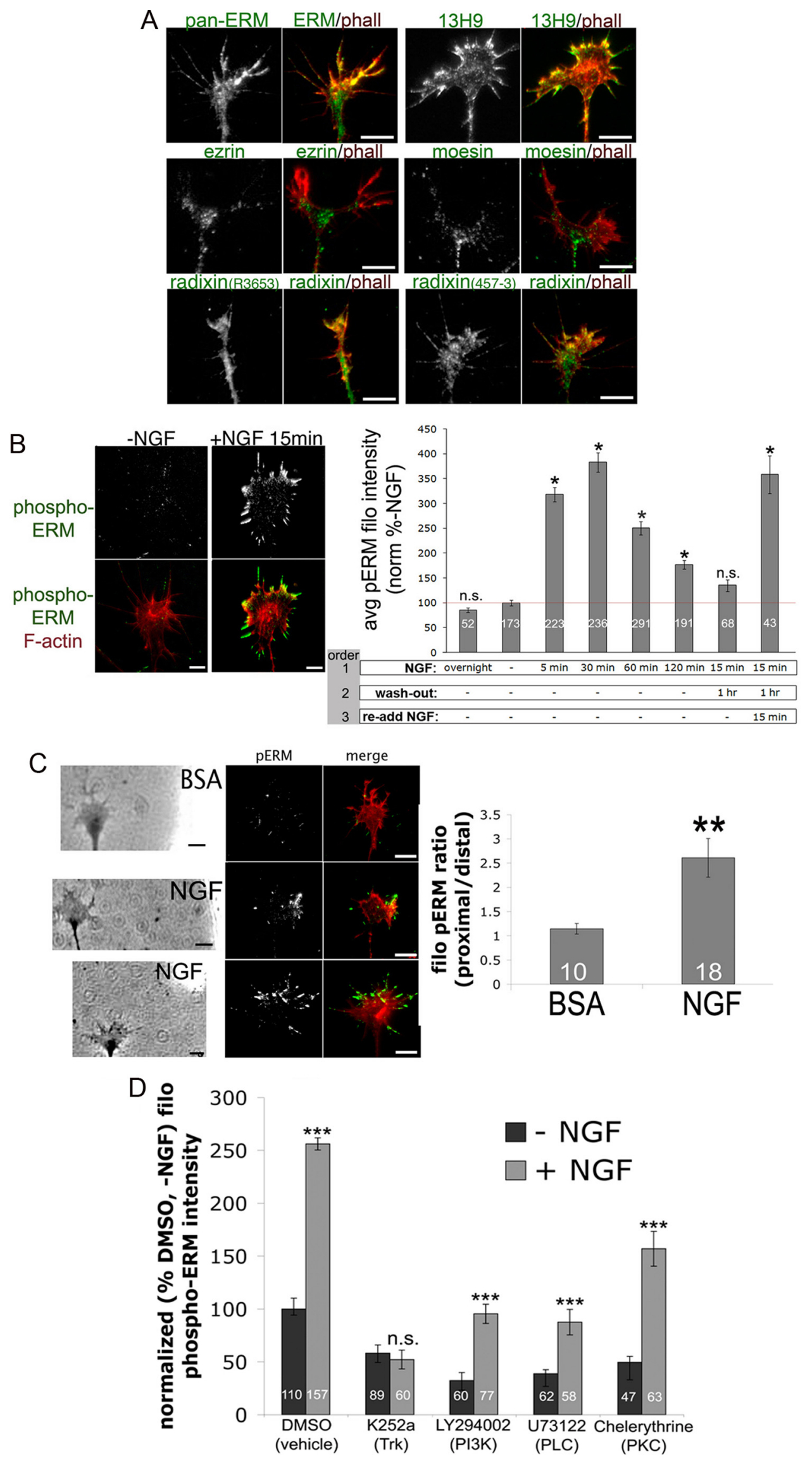

Figure 1. Nerve growth factor globally and locally activates ERM proteins. A, Embryonic day 7 (E7) DRG explants were cultured overnight on laminin with $40 \mathrm{ng} / \mathrm{ml}$ nerve growth factor, fixed, and stained with Alexa Fluor 568-phalloidin (phall; red) and antibodies against all three ERMs (pan-ERM, 13H9) or the individual ERM proteins ezrin, moesin, or radixin (R3653, 457-3). (Figure legend continues.) 
as to link a new actin polymer to the growth cone leading margin and facilitate protrusion.

Adhesive contacts enable axon growth and guidance by stabilizing protrusions, providing traction for pulling forward, and influencing intracellular signaling. Three adhesion molecules important in axon growth and guidance are L1, N-cadherin, and $\beta$-integrin dimers (Neugebauer et al., 1988; Letourneau and Shattuck, 1989; Agius et al., 1996; McKerracher et al., 1996; Cheng and Lemmon, 2004; Kiryushko et al., 2004; Chen et al., 2005; Blackmore and Letourneau, 2006a). Previous studies report that NGF treatment stimulates the accumulation of $\beta$-integrin receptors at filopodial tips (Grabham and Goldberg, 1997; Grabham et al., 2000), which we also observed in our NGFtreated DRG growth cones (Fig. $3 B, E$ ). We also report for the first time that L1 expression along filopodial shafts was significantly higher after NGF treatment (Fig. 3C,E). Interestingly, this L1 accumulation colocalized with phospho-ERM, whereas $\beta 1$ integrin accumulation at filopodial tips was distal to phosphoERM. Growth cone N-cadherin distribution was unchanged after NGF treatment, suggesting that these changes in $\beta 1$-integrin and L1 distribution are selective (Fig. 3D,E).

In Marsick et al. (2010) we showed that a gradient of NGF locally increases F-actin, free barbed ends, ADF/cofilin activity, and here, phospho-ERM (Fig. 1C). Similarly, filopodial accumulations of $\beta 1$-integrin receptors and L1 were also greater in growth cone regions proximal to an NGF source (Fig. $3 F$ ), with no such asymmetry in gradients of BSA. Thus, an NGF gradient rapidly increases actin polymerization, active ERM proteins, and adhesion receptors in the protruding growth cone region closer to the NGF source. With their dual binding functions, ERM proteins may play a key role in harnessing both newly polymerized actin filaments and adhesion molecules to effectively protrude the leading margin and lead the growth cone toward the NGF source.

\section{Disrupting ERM function disrupts growth cone F-actin and motility}

To evaluate the function of ERM proteins in DRG growth cone migration and their responses to NGF, two approaches were used. We transfected DRG neurons to express a dominantnegative ezrin mutant comprised of the membrane-binding FERM domain fused with GFP, which competes with endogenous ERM proteins and prevents actin-membrane linkage (a kind gift from Dr. Janis Burkhardt; described by Allenspach et al., 2001). Alternatively, we transfected DRG neurons to express a mixture of siRNA against radixin and moesin. Knockdown of radixin and moesin protein levels by siRNA was confirmed by Western blot (Fig. 4A) and immunocytochemistry (Fig. 4B, C).

(Figure legend continued.) $\quad \boldsymbol{B}$, E7 DRG explants were cultured overnight without NGF (except in the first bar, $40 \mathrm{ng} / \mathrm{ml} \mathrm{NGF} \mathrm{overnight),} \mathrm{and} \mathrm{then} \mathrm{NGF} \mathrm{(40} \mathrm{ng/ml)} \mathrm{was} \mathrm{bath} \mathrm{applied} \mathrm{for} \mathrm{the}$ times indicated, washed out as indicated, and cells were fixed, stained for F-actin (phall; red) and phospho-ERM (green), and filopodial (filo) phospho-ERM intensities were measured. C, A BSA- or NGF-coated micropipette was lowered to one side of a growth cone for $5 \mathrm{~min}$ and then cells were fixed and stained for F-actin (phalloidin; red) and phospho-ERM (green). The ratio of filopodial phospho-ERM distribution was measured as proximal/distal intensity relative to NGF source. D, DRG cultures were treated for 15 min with DMSO, 500 nм K252a, $10 \mu \mathrm{m} \mathrm{LY294002,} 1$ $\mu \mathrm{M}$ U73122, or $1 \mu \mathrm{m}$ chelerythrine, treated with NGF for $10 \mathrm{~min}$, and then fixed. Filopodial phospho-ERM staining intensity was quantified and normalized to the DMSO-NGF condition. Statistical significance was determined using Student's $t$ test. Data are means $\pm \mathrm{SEM}$; ${ }^{*} p<$ $0.05,{ }^{* *} p<0.001,{ }^{* * *} p<0.001$. Scale bars, $10 \mu \mathrm{m}$. avg, Average.
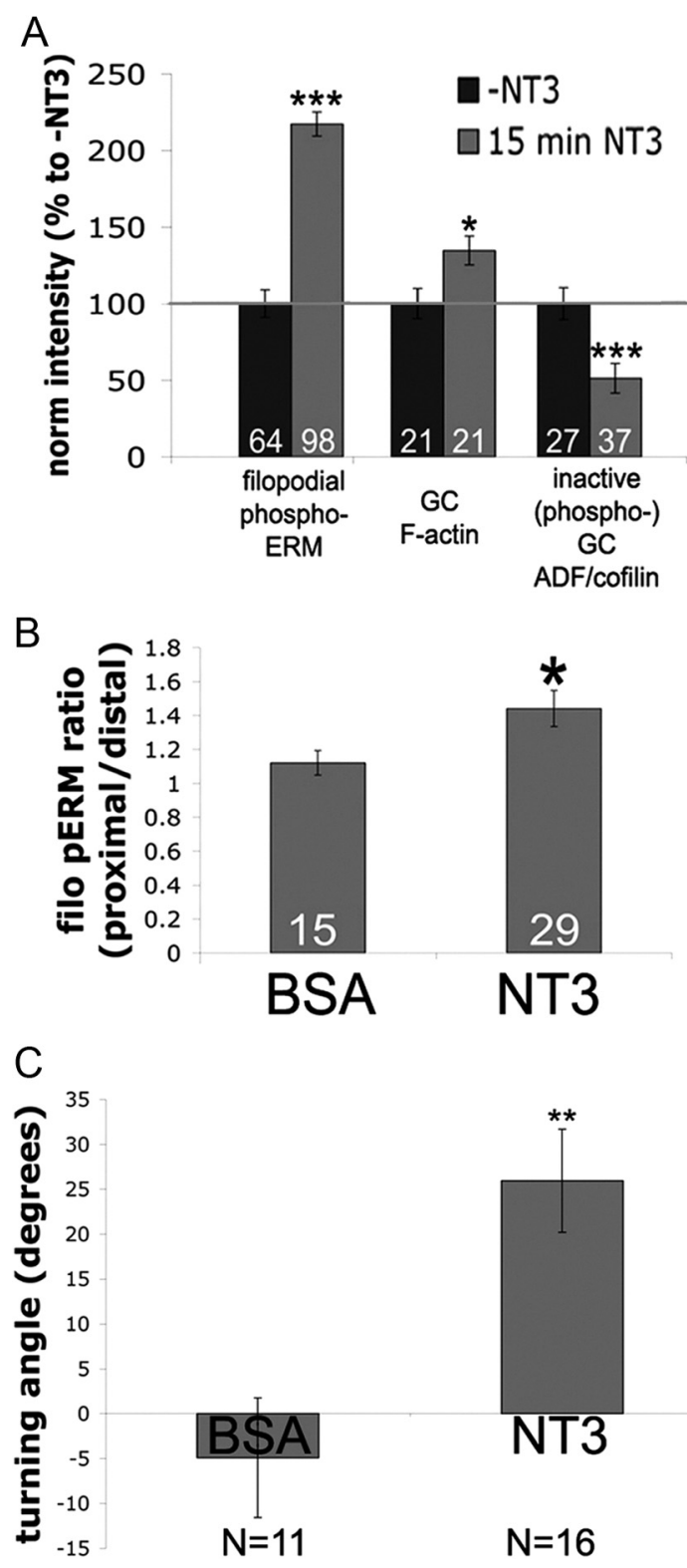

Figure 2. Attractive guidance cue neurotrophin-3 activates ERM proteins. $\boldsymbol{A}, \mathrm{DRG}$ explants cultured overnight and treated $15 \mathrm{~min}$ with $40 \mathrm{ng} / \mathrm{ml} \mathrm{NT3}$ and fixed and stained for phosphoADF/cofilin, F-actin (phalloidin), or phospho-ERM. B, A BSA- or NT3-coated micropipette was lowered to one side of a growth cone for $5 \mathrm{~min}$ and then cells were fixed and stained for $F$-actin and phospho-ERM. The ratio of filopodial (filo) phospho-ERM distribution was measured as proximal/distal intensity relative to the NT3 source. C, DRG explants were growth overnight with $0.3 \mathrm{ng} / \mathrm{ml} \mathrm{NT3}$. Growth cones were imaged for $15 \mathrm{~min}$, subjected to NT3 diffusing from a micropipette to one side and imaged for an additional $45 \mathrm{~min}$, and then mean turning angles were measured. Statistical significance was determined using Student's $t$ test. Data are means \pm SEM; ${ }^{*} p<0.05,{ }^{* *} p<0.001,{ }^{* * *} p<0.0001$.

To assess how disrupting ERM function affects growth cone actin organization, we used confocal microscopy to visualize phalloidin-labeled F-actin. In GFP controls, F-actin is organized in peripheral filopodial bundles and lamellipodial structures and is less prevalent in the $\mathrm{C}$-domain where filaments are disassembled (Fig. $4 D, E$ ). In contrast, DN-ERM-expressing growth cones exhibited less F-actin in the P-domain, with F-actin tangles more centrally located (Fig. 4D,E). This might occur because without ERM-mediated linkage to plasma membrane proteins, F-actin is more readily transported retrogradely from the $\mathrm{P}$-domain into the C-domain. We probed this possibility by co-transfecting neu- 

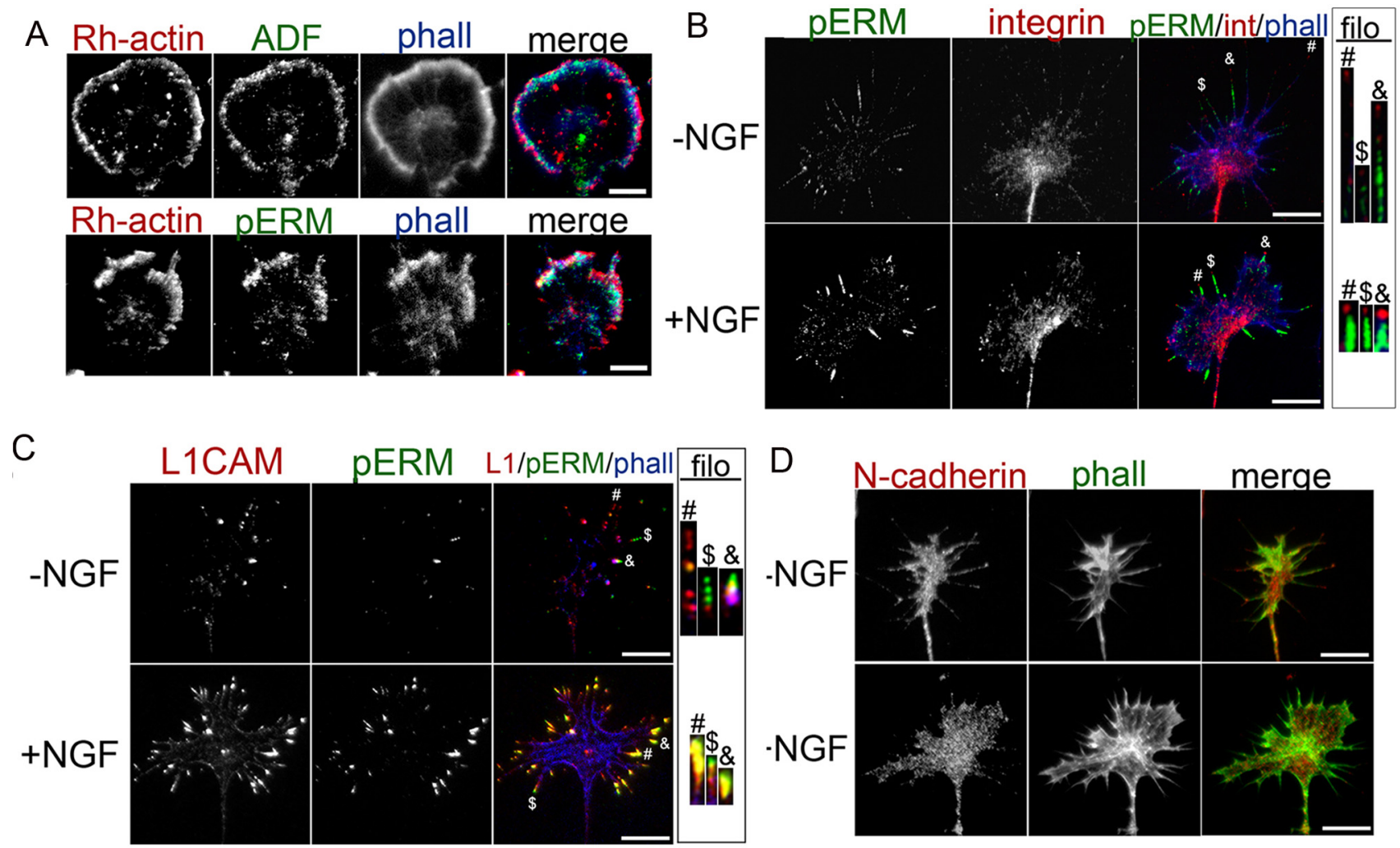

E
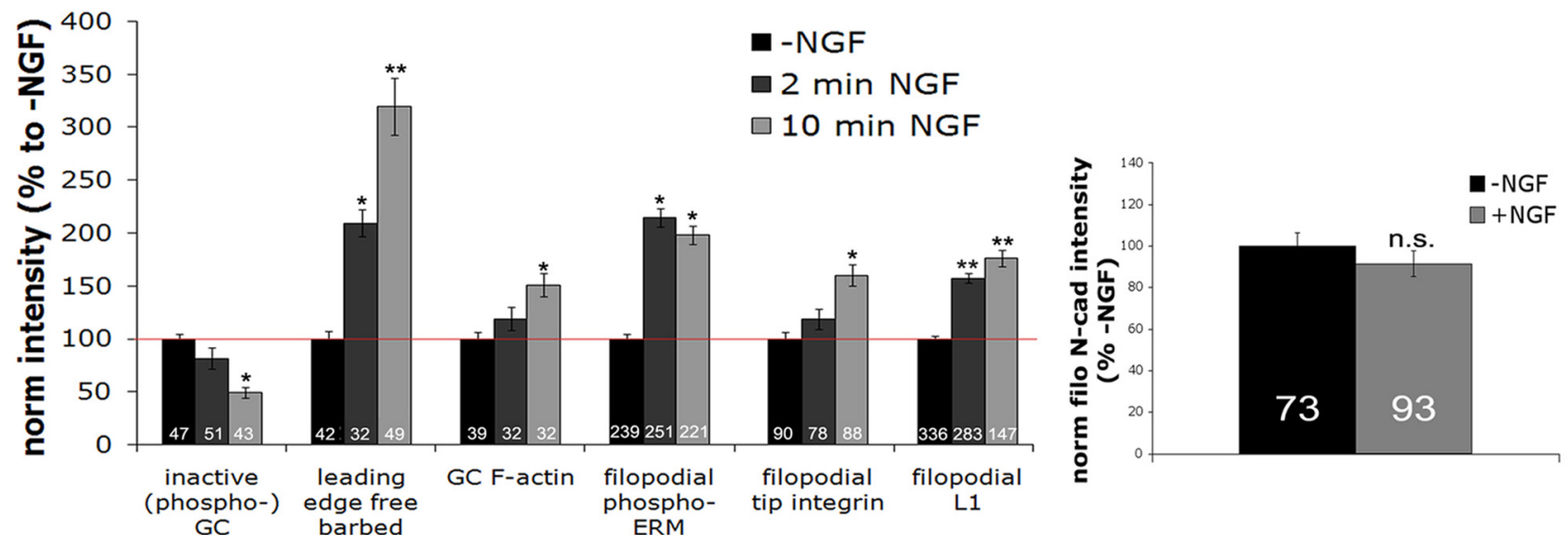

panel:

(A) $\quad(A, B, C) \quad(B, C)$

(B)

(C)

(D)

F
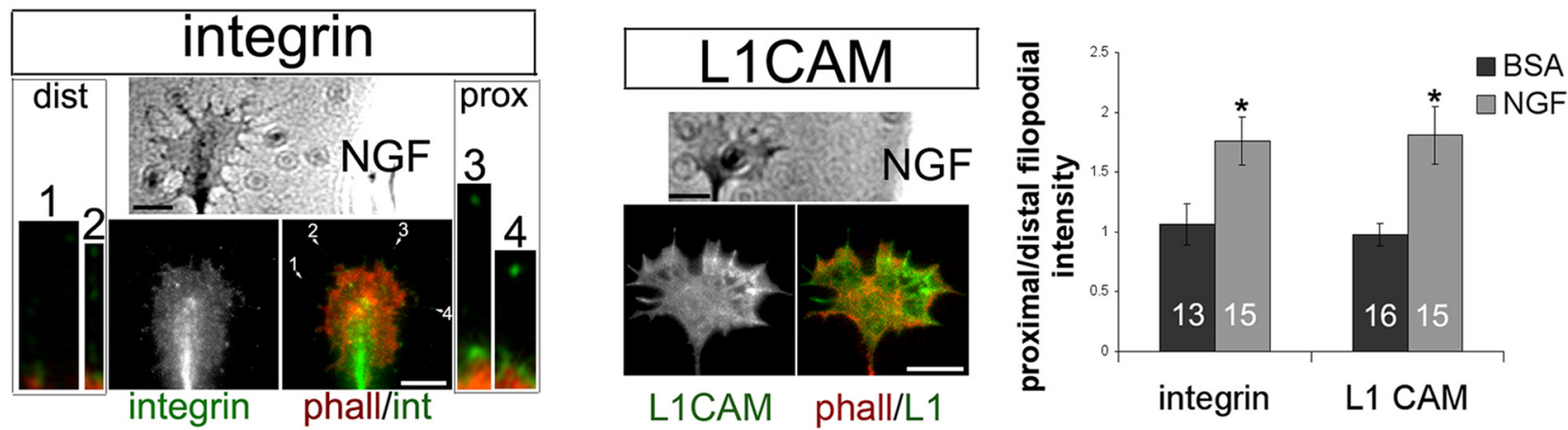

Figure 3. NGF treatment colocalizes phospho-ERM and ADF/cofilin at the leading edge and increases filopodial L1 and $\beta$-integrin expression. DRG explants were cultured overnight without NGF and treated for 10 min with $40 \mathrm{ng} / \mathrm{ml} \mathrm{NGF.} \mathrm{A,} \mathrm{For} \mathrm{the} \mathrm{ADF} \mathrm{condition,} \mathrm{cells} \mathrm{were} \mathrm{permeabilized} 1 \mathrm{~min}$ with Alexa Fluor 350-phalloidin (phall; blue), Rh-actin labeling was performed for 4 min (red), and then cells were fixed and stained for ADF (ab12977; green). For the phospho-ERM condition, cells were permeabilized for 1 min with buffer containing Alexa Fluor 350-phalloidin (blue), 0.1\% glutaraldehyde, and $0.1 \%$ paraformaldehyde. Rh-actin labeling was performed for $4 \mathrm{~min}$ (red) and then cells were further fixed for $30 \mathrm{~min}$ and stained for phospho-ERM (green). $\boldsymbol{B}$ - $\boldsymbol{D}, \mathrm{DRG}$ explants

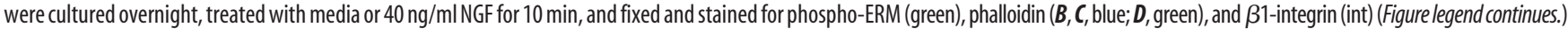


rons to express DN-ERM-GFP or control GFP, as well as RFPactin (Chan and Odde, 2008; Marsick et al., 2010). By tracking movement of bright red actin features, we found increased retrograde flow of F-actin at the leading margin of DN-ERMexpressing growth cones compared to control GFP (Fig. 4F). Here, reduced ERM-mediated membrane linkage may facilitate retrograde F-actin flow, disrupting F-actin organization in the peripheral domain. In support of this we found lower growth cone F-actin levels (Fig. 4G) and fewer filopodia (Fig. 4H) in DN-ERM growth cones, suggesting that ERM-mediated linkage of actin to membrane proteins supports the establishment and/or maintenance of the F-actin network in the motile periphery.

To examine the effects of disrupted ERM function on motility, we used time-lapse imaging to assess the rate of growth cone advance. Compared to GFP-expressing controls, DN-ERM growth cones advanced more slowly (Fig. $4 J$ ) and, not surprisingly, axons of DN-ERM-expressing neurons were shorter than GFP controls after $48 \mathrm{~h}$ in vitro (Fig. 4 I). Together, these data suggest that ERM proteins are important for the establishment and/or maintenance of actin filament organization in the growth cone leading margin.

\section{Disrupting of ERM function impairs DRG responses to NGF}

We next asked how growth cone responses to NGF are affected when ERM proteins are disrupted. Imaging was conducted for 60 min after introduction of the pipette to allow enough time for slower-moving DN-ERM- and siRNA-expressing growth cones to advance at least $80 \mu \mathrm{m}$ and execute a turning response. In both cases of reduced ERM function, siRNA treatment, or DN-ERM expression, the turning of DRG growth cones toward a micropipette releasing NGF was significantly reduced compared to GFPexpressing controls (Fig. 5A).

We previously showed that acute NGF exposure increases growth cone F-actin and decreases G-actin levels (Marsick et al., 2010), so we examined the effects of ERM inhibition on NGFstimulated actin polymerization. When NGF was added globally to DRG neurons, the increase in total F-actin seen in control GFP-expressing neurons was absent in DN-ERM-expressing growth cones (Fig. 5B).

Because the NGF-induced increase in F-actin is mediated by $\mathrm{ADF} /$ cofilin activation, we wondered if this effect of DN-ERM expression on F-actin levels was due to a lack of $\mathrm{ADF} /$ cofilin activation. However, NGF-induced activation of ADF/cofilin, as indicated by reduced staining for the inactive phospho-ADF/cofilin (pAC), was still observed in DN-ERM-expressing growth cones (Fig. 5C). Similarly, when ADF/cofilin activity in growth cones was directly increased by Chariot-mediated incorporation of constitutively active ADF/cofilin (Gehler et al., 2004; Marsick et al., 2010), DN-ERM-expressing growth cones did not exhibit the expected F-actin increase (Fig. 5D). Thus, the increased ADF/ cofilin activity that normally triggers actin polymerization at the growth cone leading margin may be ineffective without ERM function to harness actin filaments at the leading edge.

The NGF-induced increase in ADF/cofilin activity in DNERM growth cones (Fig. 5C) suggests that impaired ERM func-

\section{$\leftarrow$}

(Figure legend continued.) $\quad(\boldsymbol{B}$, red), $\mathrm{L} 1(\boldsymbol{C}$, red $)$, or N-cadherin $(\boldsymbol{D}$, red). $\boldsymbol{E}$, Intensity measurements were performed as described in Materials and Methods. $\boldsymbol{F}, \mathrm{A}$ BSA- or NGF-coated micropipette was lowered to one side of a growth cone for $5 \mathrm{~min}$ and then cells were fixed and stained for phalloidin (red) and $\beta$-integrin or L1 (green). The ratio of filopodial $\beta$-integrin or L1 intensity distribution was measured as proximal (prox)/distal (dist) relative to pipette source. For insets at integrin image, filopodia were enlarged $300 \%$. Statistical significance was determined using Student's $t$ test. Data are means \pm SEM; ${ }^{*} p<0.05,{ }^{* *} p<0.01$. Scale bars, $10 \mu \mathrm{m}$. tion does not block NGF signaling. To more directly probe this, we stained GFP and DN-ERM growth cones with an antibody against the high-affinity NGF receptor TrkA. DN-ERM growth cones did express TrkA, although with lower levels compared to control GFP growth cones (Fig. $5 E$ ). We therefore sought to assess whether this reduced level of TrkA expression impaired DNERM-expressing growth cones' ability to detect NGF and activate downstream signaling. We thus used an antibody against phosphoAkt, an activated downstream target of NGF signaling (Crowder and Freeman, 1998). As expected, NGF exposure increased phospho-Akt levels in GFP-expressing growth cones (Fig. 5F). Inhibition of PI3K (LY-294002), which has been implicated in ERM activation (Gautreau et al., 1999; Gallo, 2008; Jeon et al., 2010) and lies upstream of TrkA-mediated Akt activation, blocks this increase (Fig. 5F). However, DN-ERM-expressing growth cones also had significantly increased levels of phospho-Akt following NGF (Fig. 5F), suggesting that disrupting ERM function does not interfere with NGF detection and signaling.

Thus, impaired ERM function blocks the NGF-induced increase in actin polymerization, despite evidence that TrkAmediated signaling occurs and ADF/cofilin is activated. Even a direct increase in $\mathrm{ADF} /$ cofilin activity, independently of NGF signaling, failed to increase F-actin in DN-ERM-expressing growth cones (Fig. 5D). These data suggest that ERM-mediated F-actin-membrane linkage is required for increased ADF/cofilin activity to trigger actin polymerization at the leading margin.

\section{Disruption of ERM function disrupts NGF-induced increase in active $\mathrm{ADF} /$ cofilin, F-actin barbed ends, and adhesion proteins at the leading growth cone margin}

Thus far, we have found that NGF does not increase F-actin levels in DN-ERM-expressing growth cones, despite ADF/cofilin activation. To further explore the effects of disrupting ERM function, we visualized the distribution of active ADF/cofilin and F-actin barbed ends in NGF-stimulated growth cones. To enable identification of transfected cells following the permeabilization step of barbed-end labeling, we cotransfected neurons to express GFP or DN-ERM and GFP-actin, which incorporates into detergentresistant actin filaments. Following acute NGF treatment, active $\mathrm{ADF} /$ cofilin normally localizes to the growth cone leading margin, where it severs filaments to create free F-actin barbed ends (Figs. 3A, 6A; Marsick et al., 2010). However, as shown in Figure $6 A$, active $\mathrm{ADF} /$ cofilin is more centrally located in DN-ERMexpressing growth cones.

We then probed whether NGF-induced free barbed ends are also displaced when ERM function is disrupted. In growth cones transfected with GFP-actin and control GFP, most rhodamineactin was incorporated at the leading margin, consistent with the localization of $\mathrm{ADF} /$ cofilin (Fig. $6 \mathrm{~B}, \mathrm{C}$ ). However, in growth cones that also expressed DN-ERM, more rhodamine-actin was incorporated centrally and away from the leading margin, where the active ADF/cofilin was also mislocalized in the NGF-treated DN-ERM-expressing growth cones (Fig. 6B,C).

Together, these experiments suggest that ERM proteins play a critical role in attractive responses to growth cone guidance cues. When ERM function is reduced, F-actin is not organized into the typical peripheral bundles and networks. Rather, lamellipodia are absent and more F-actin is centrally concentrated, perhaps because retrograde actin flow is enhanced without ERM-mediated F-actin linkage to the plasma membrane (Fig. $4 F$ ). Upon NGF treatment total F-actin content is not increased, although ADF/ cofilin activity and F-actin barbed ends are increased, as normal. However, these components are mislocalized centrally in DN-ERM 
A
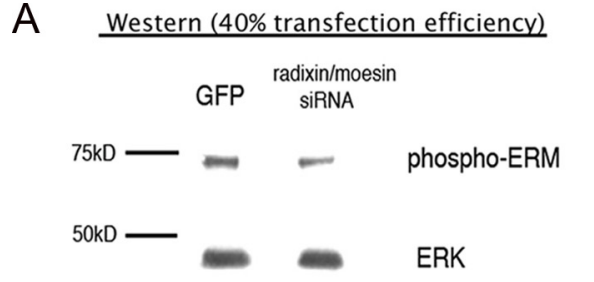

B

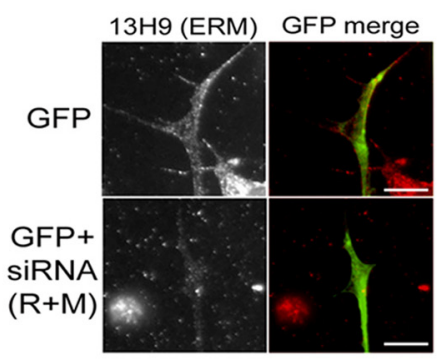

$\mathrm{C}$

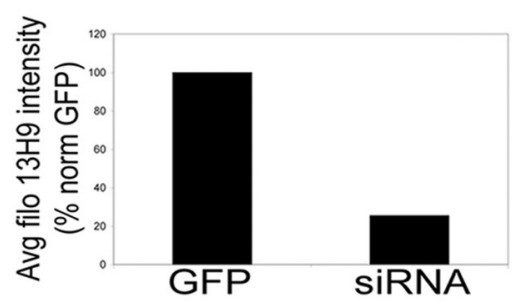

D

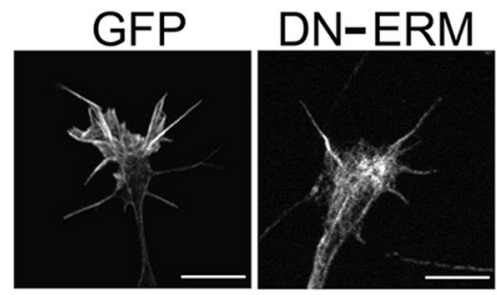

E

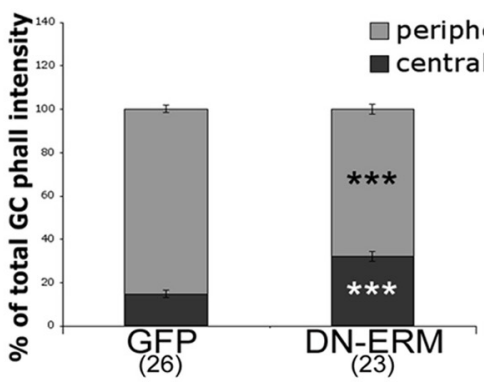

$\mathrm{F}$

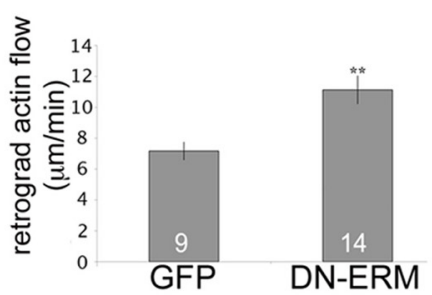

G

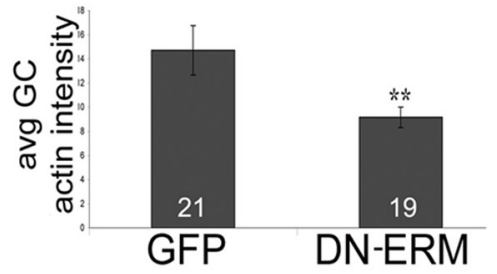

$\mathrm{H}$
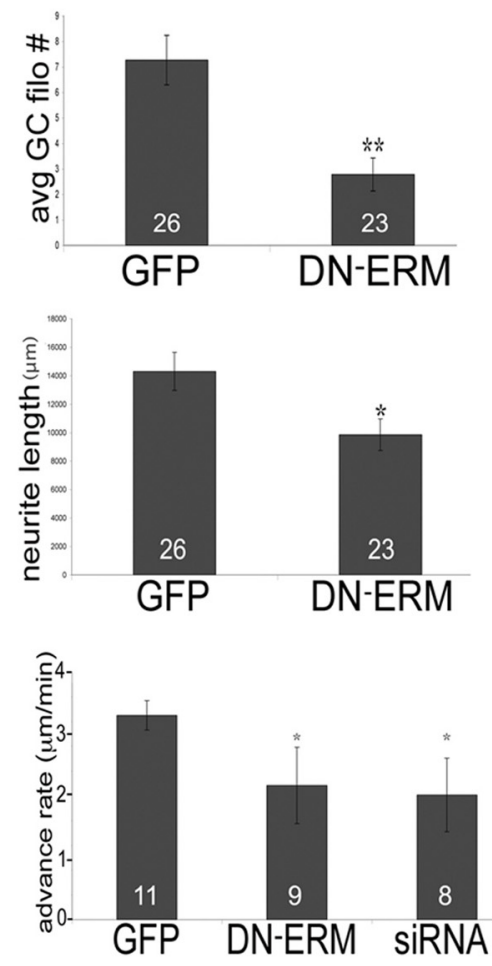

Figure 4. Disrupting ERM function results in smaller and less motile growth cones with disorganized actin filaments. DRGs were transfected with GFP control or siRNA against radixin and moesin $(R+M)$ and cultured for $48 \mathrm{~h}$ with $40 \mathrm{ng} / \mathrm{ml} \mathrm{NGF.} \boldsymbol{A}-\boldsymbol{C}$, Cells were lysed and probed for phospho-ERM via Western blot $(\boldsymbol{A})$ or fixed and stained with an antibody against ERMs (13H9) $(\boldsymbol{B})$, and intensities were quantified (C). D, DRGs were transfected with GFP or DN-ERM, cultured overnight with NGF, fixed, stained with phalloidin, and imaged using confocal microscopy. $\boldsymbol{E}-\boldsymbol{H}$, The following parameters were quantified: phalloidin intensities in the peripheral and central growth cone $(\mathrm{GC})$ regions $(\boldsymbol{E})$, growth cone actin intensity $(\boldsymbol{G})$, or filopodial number $(\boldsymbol{H})$. For $\boldsymbol{F}$, DRGs were cotransfected with RFP-actin and GFP or DN-ERM and cultured overnight. Growth cones were imaged for 6 min at 3 s intervals and bright RFP-actin features were tracked to calculate retrograde flow rates. I, Neurite lengths were measured for GFP and DN-ERMexpressing DRGs cultured overnight with NGF. J, GFP, DN-ERM, or siRNA transfected growth cones were imaged every min for $1 \mathrm{~h}$ to calculate advance rate. Statistical significance was determined using Student's t test. Data are means $\pm \mathrm{SEM} ;{ }^{*} p<0.05,{ }^{* *} p<$ $0.01,{ }^{* *} p<0.001$ Scale bars, $10 \mu \mathrm{m}$. Avg/avg, Average.

growth cones. Thus, ERM proteins may contribute to an attractive response by organizing actin filaments at the leading edge to allow an effective protrusion response and by tethering newly created barbed ends to the plasma membrane for sustained actin polymerization.

We previously showed that NGF treatment increases filopodial expression of the adhesion molecules L1 and $\beta 1$-integrin (Fig. $3 B, C, E)$. These increases were also blocked when ERM function was disrupted (Fig. 6D,E,F). The absence of $\mathrm{L} 1$ accumulation is likely related to a reduction in ERM-mediated linkage of L1 to filopodial F-actin, given that L1 and ERMs can physically interact (Dickson et al., 2002; Cheng et al., 2005) and that L1 colocalizes with active phospho-ERM in filopodia (Fig. 3C). However, the reduced $\beta 1$-integrin localization in filopodial tips may reflect other consequences of the disrupted F-actin arrays, given that $\beta 1$-integrin does not colocalize with phospho-ERM in filopodia (Fig. $3 B$ ). Finally, DN-ERM did not affect N-cadherin distribution (Fig. 6G), whose distribution is not affected by NGF treatment (Fig. 4D). Thus, the ability of NGF to induce changes in the distribution and organization of adhesion-mediating proteins on filopodia also depends on ERM function.

Increased ADF/cofilin activity increases the levels of phospho-ERM

An early biochemical study suggested that radixin binds preferentially to $\mathrm{F}$-actin barbed ends (Tsukita et al., 1989). We have shown here that phospho-ERM, $\mathrm{ADF} /$ cofilin, and barbed ends colocalize at the leading growth cone margin following NGF treatment (Fig. 3A). We therefore wondered if the increased phospho-ERM levels following NGF might be due, at least in part, to the increased free F-actin barbed ends created by ADF/cofilin activity. In a previous study we showed that without adding NGF, a direct increase in growth cone $\mathrm{ADF} /$ cofilin activity increases free barbed ends (Marsick et al., 2010). Using this method, we introduced constitutively active ADF/cofilin (A3) into cultured DRGs using Chariot for $1 \mathrm{~h}$ and then fixed and assessed phospho-ERM levels. We found that similar to treatment with NGF, growth cones with elevated ADF/ cofilin (char + A3) also exhibited increased filopodial phospho-ERM staining, while no such increase occurred with chariot + BSA controls (Fig. 7A). In Marsick et al. (2010) we also found that a gradient of cellpermeable, active ADF/cofilin (chariot + A3) was sufficient to locally increase growth cone F-actin and free barbed ends, as well as attractive growth cone turning toward the region of higher $\mathrm{ADF} /$ cofilin activity. We therefore subjected growth cones to this chariot $+\mathrm{A} 3$ gradient for $5 \mathrm{~min}$ before being fixed and stained for phospho-ERM. While a gradient of chariot + BSA resulted in symmetric phosphoERM staining across growth cone widths, chariot $+\mathrm{A} 3$ gradients produced increased phospho-ERM closest to the source (Fig. $7 B$ ).

To determine whether reducing $\mathrm{ADF} /$ cofilin activity conversely lowers phospho-ERM levels, we transfected DRG neurons with a constitutively active form of LIM kinase (CA-LIMK) that phosphorylates and inactivates ADF/cofilin (Yang et al., 1998; 
A

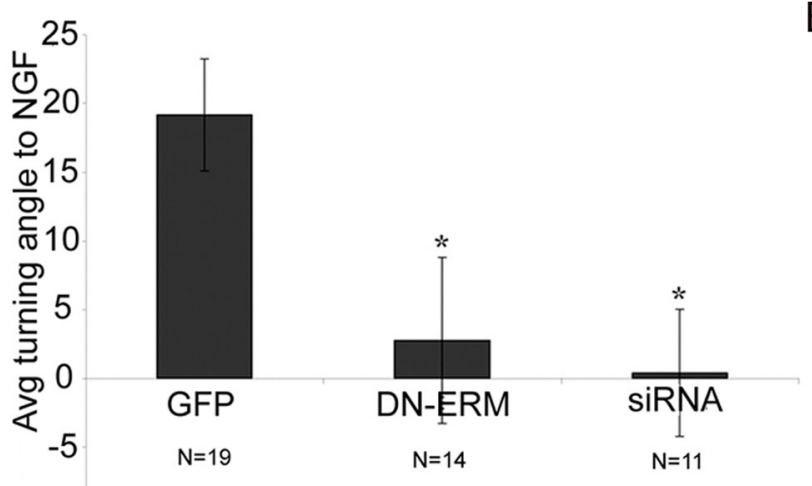

$-10$

C

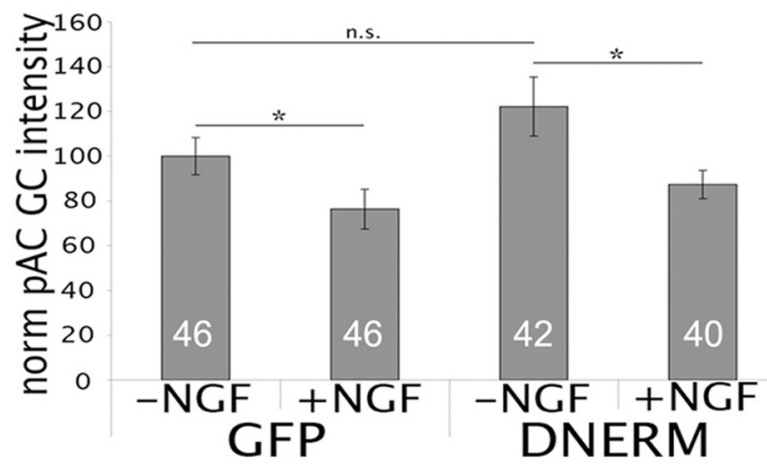

B

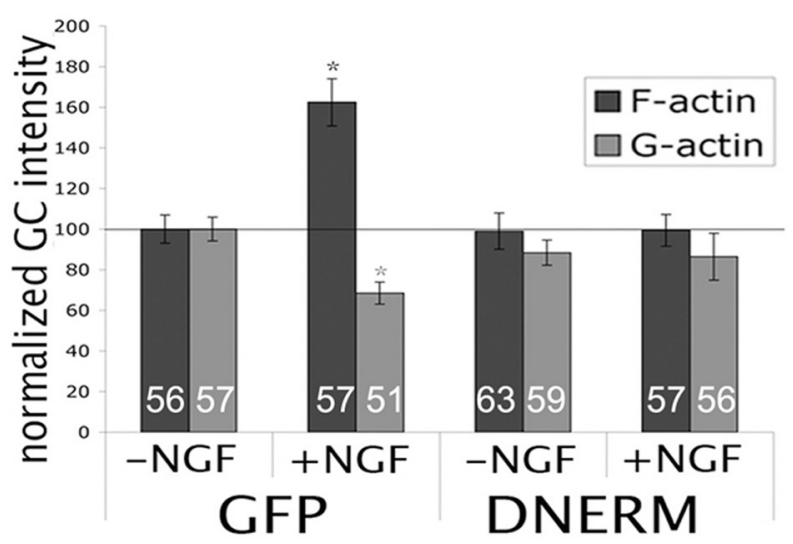

$D$

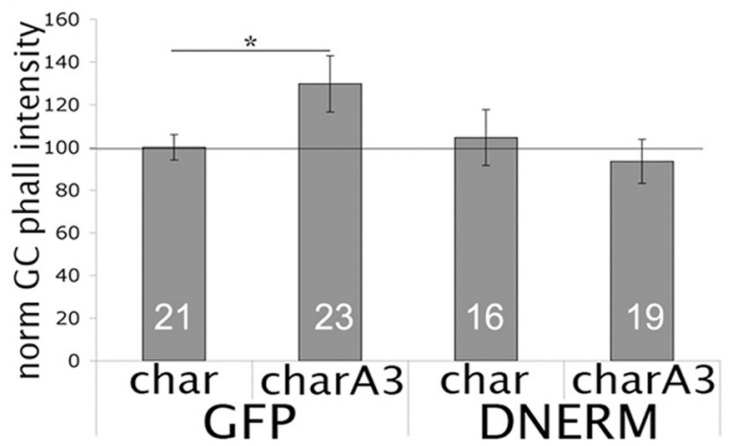

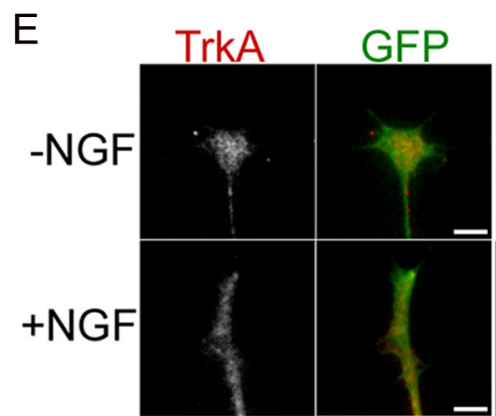

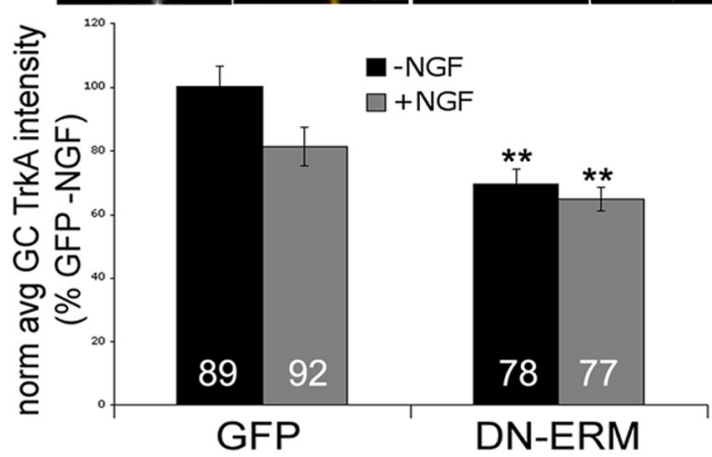

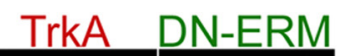

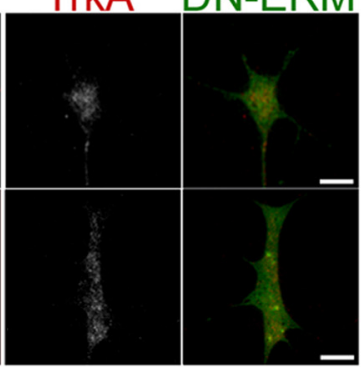

F
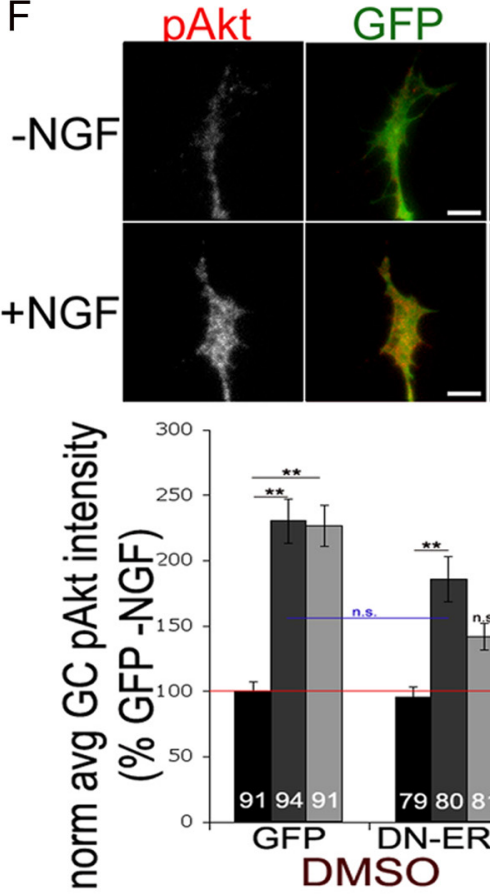

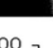

]

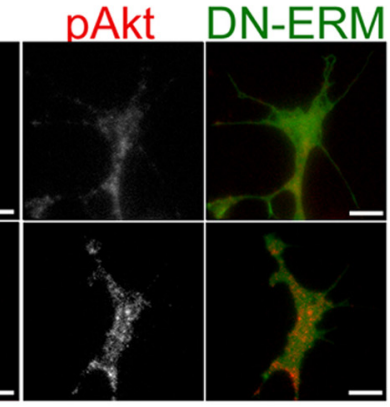

-NGF

$\square N G F 5 \mathrm{~min}$

$\square N G F 20 \mathrm{~min}$

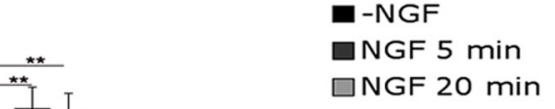


A
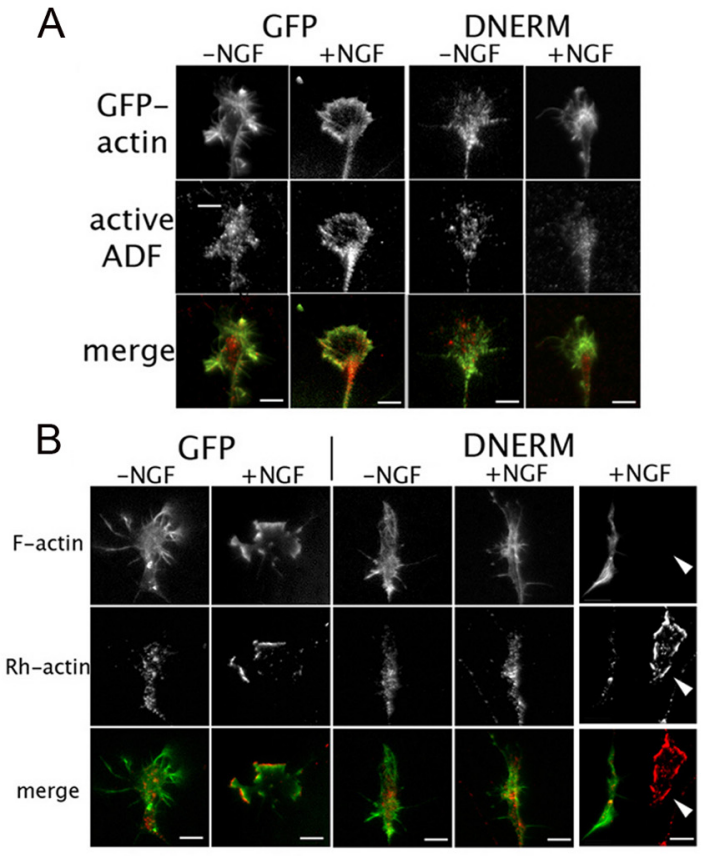

C

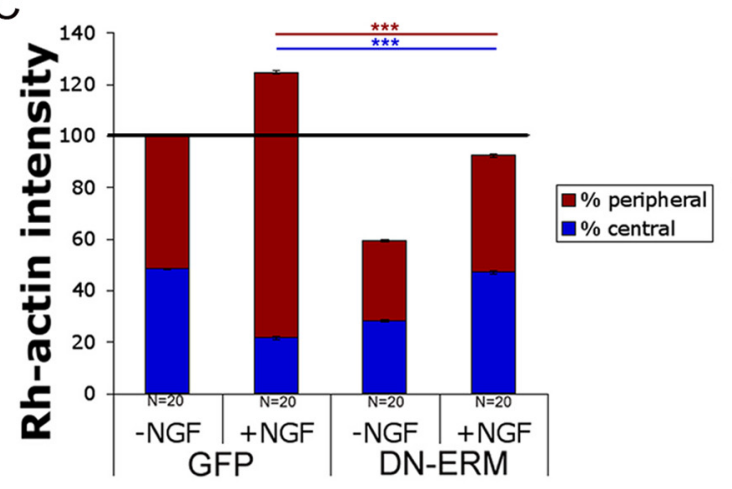

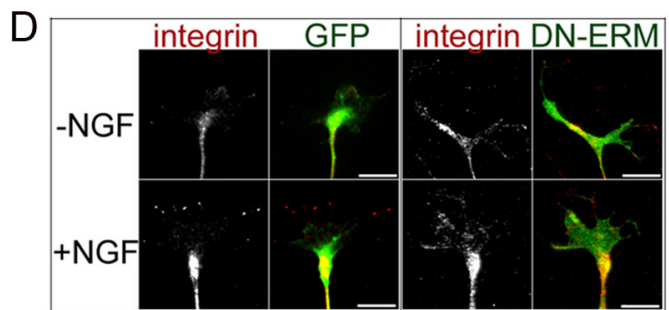

E

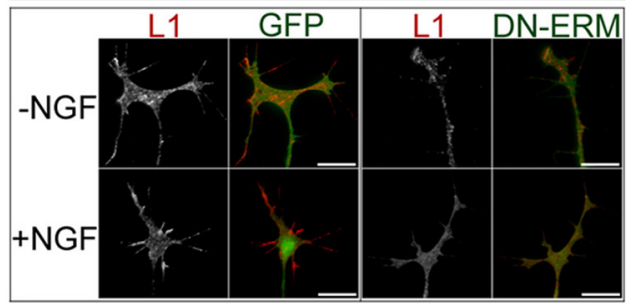

F

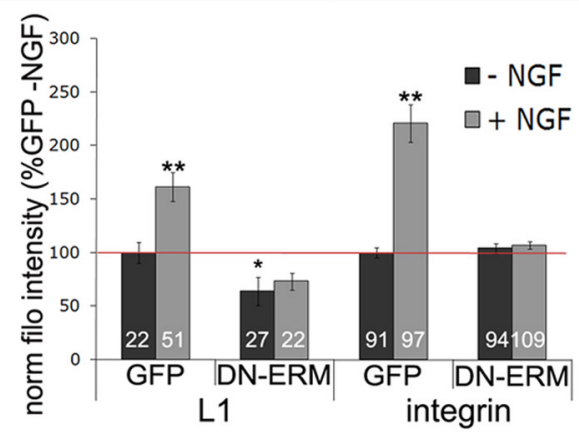

G

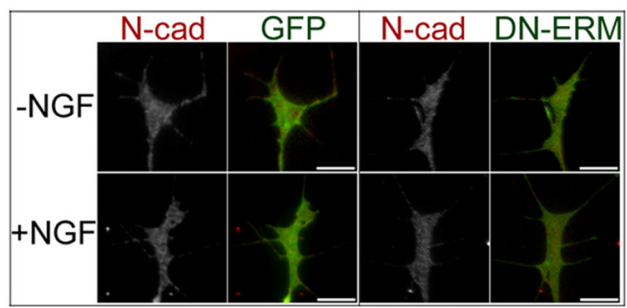

Figure 6. Disrupting ERM function disrupts NGF-induced increase in leading edge active ADF/cofilin and F-actin barbed ends, and filopodial L1 and $\beta$-integrin. DRGs were transfected with GFP or DN-ERM, cultured overnight, and treated for $15 \mathrm{~min}$ with medium or $40 \mathrm{ng} / \mathrm{mI} \mathrm{NGF.} A$, Cell were permeabilized for $1 \mathrm{~min}$ and then fixed and stained with an antibody against total ADF (12977). $B-C$, Cells were permeabilized for $1 \mathrm{~min}$, barbed end labeling was performed for $4 \mathrm{~min}$, and then cells were fixed and imaged. Barbed end labeling quantification in peripheral versus central regions was performed as described in Materials and Methods. Arrowheads in $\boldsymbol{B}$ mark an untransfected growth cone in DN-ERM culture that shows normal Rh-actin barbed end labeling $\boldsymbol{D}-\mathbf{G}$, Cells were fixed and stained for $\beta 1$-integrin (D), L1 (E), or N-cadherin (N-cad) (G). Intensities of $\mathrm{L} 1$ in filopodia (filo) or $\beta 1$-integrin at filopodial tips were measured $(\boldsymbol{F})$. Statistical significance was determined using Student's $t$ test. Data are means \pm SEM; ${ }^{*} p<0.05,{ }^{* *} p<0.01,{ }^{* * *} p<0.001$. Scale bars, $10 \mu \mathrm{m}$.

Bernard, 2007). Neurons expressing CA-LIMK do not exhibit an NGF-induced increase in F-actin (Marsick et al., 2010). Compared to GFP controls, CA-LIMK-expressing growth cones did not demonstrate a NGF-induced increase in filopodial phosphoERM (Fig. 7C).

This finding of increased phospho-ERM levels following a direct increase in ADF/cofilin activity and free F-actin barbed ends, without adding NGF, suggests that ERM proteins may be activated or stabilized by binding to new F-actin barbed ends at the plasma membrane. In this sense, activation of ADF/cofilin lies upstream of ERM activation in growth cones responding to NGF signaling.

\section{Filopodial phospho-ERM expression is reduced by}

\section{L1 knockdown}

ERM proteins can stabilize peripheral growth cone F-actin via N-terminal linkage to cytoplasmic domains of membrane proteins. Thus far we have shown that L1 and active ERM proteins colocalize in filopodia, and previous studies have shown direct interaction of these proteins in neurons (Dickson et al., 2002; Cheng et al., 2005). These data suggested to us that L1 may often participate with ERM proteins in forming the membrane link that helps to organize dynamic actin-rich growth cone structures. To test this possibility, we reduced expression of L1 by electroporating an anti-L1 FITC-tagged morpholino into DRG neurons. As shown in Blackmore and Letourneau (2006b), DRG explants treated with L1 morpholino (L1 MO) do not extend neurites on L1, but they do on laminin. Also, as expected (Fig. $8 \mathrm{~A}$ ), growth cones treated with $\mathrm{L} 1 \mathrm{MO} 48 \mathrm{~h}$ in vitro demonstrate reduced staining for L1 compared to controls. To assess F-actin organization when L1 levels are reduced, we imaged phalloidin-labeled F-actin in morpholino-treated growth cones. Similar to DNERM-expressing growth cones, growth cones with reduced L1 were smaller (Fig. $8 \mathrm{~B}$ ) and had fewer filopodia (Fig. 8C). In addition, after 48 of knockdown of L1 growth cone migration was greatly reduced, with many growth cones making little to no progress (Fig. $8 \mathrm{D}$ ). As predicted by our suggestion, growth cones with reduced L1 expression also exhibited reduced phospho- 
A
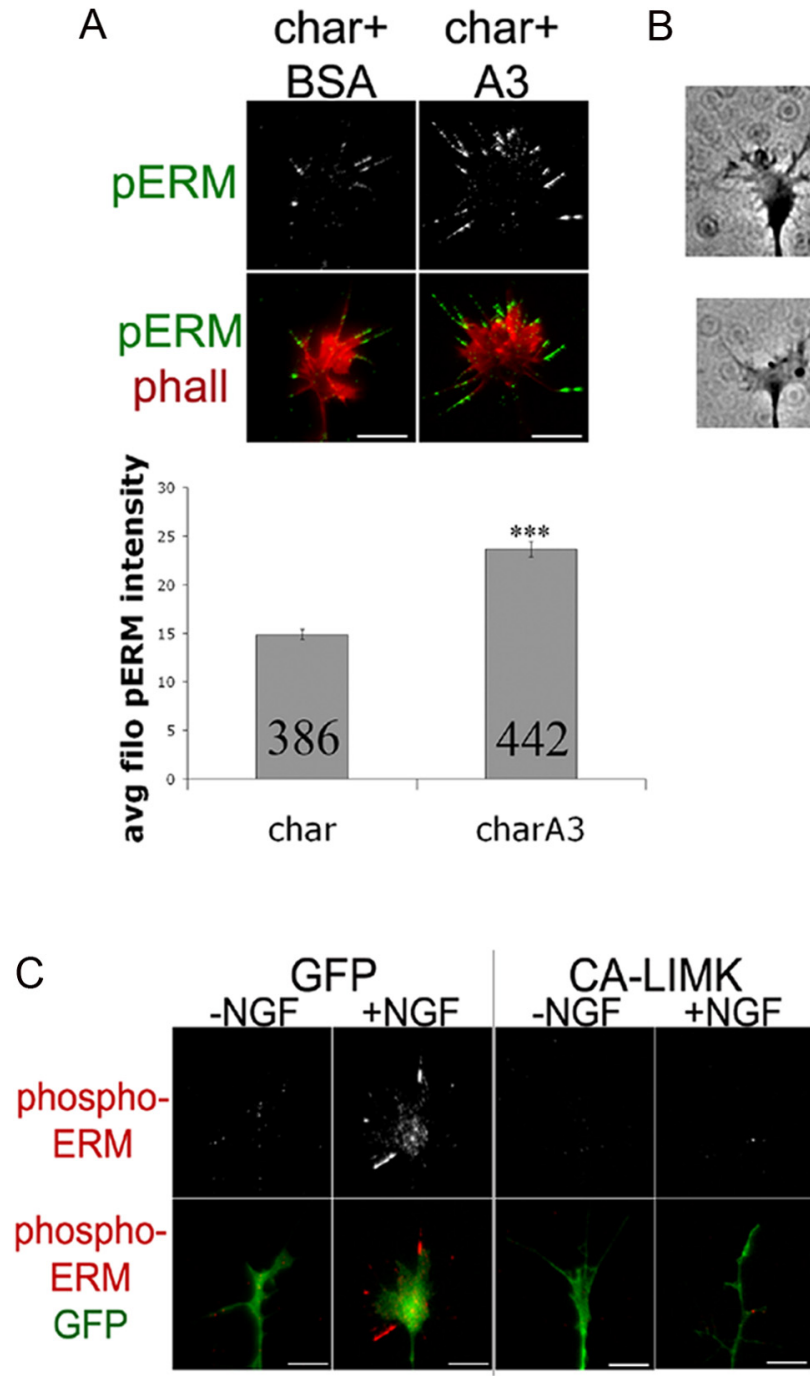

B
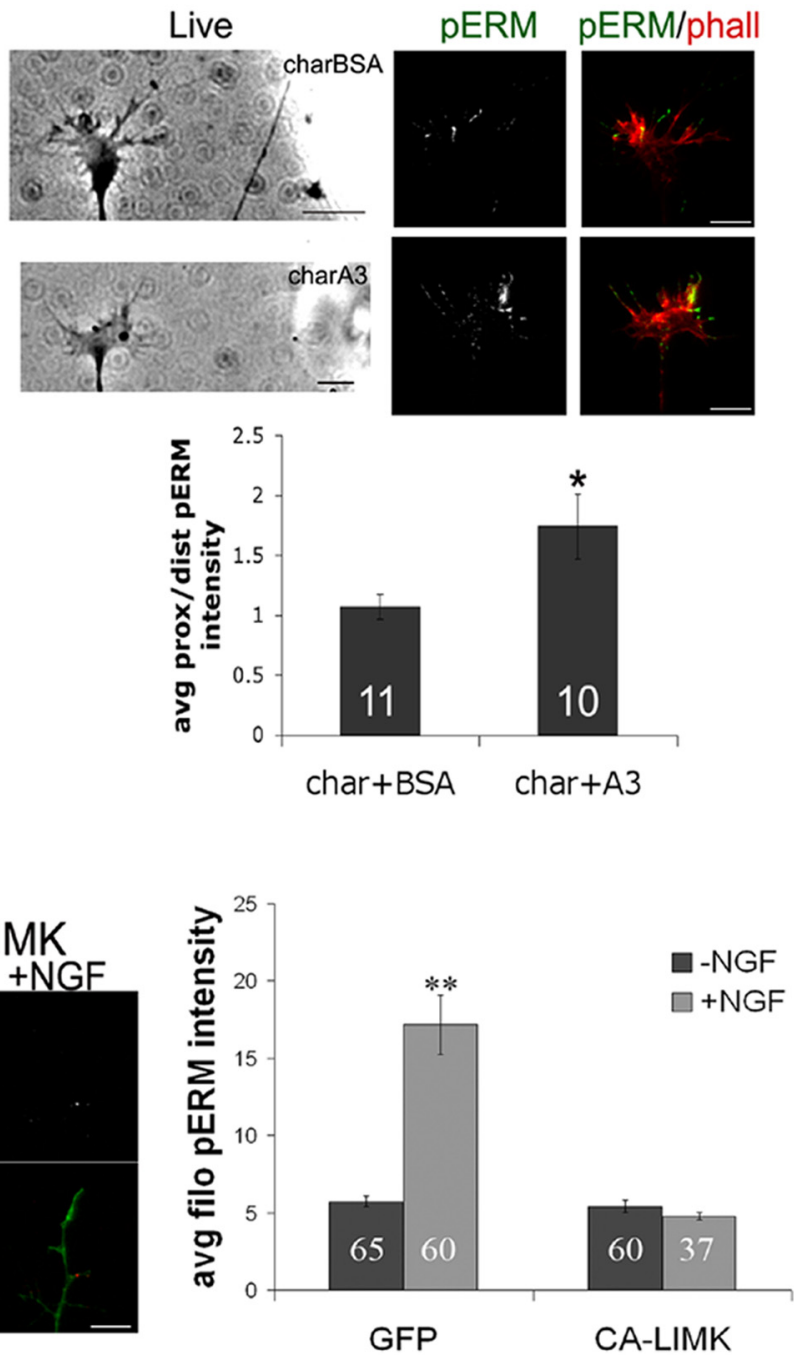

Figure 7. Increasing ADF/cofilin activity increases phospho-ERM. DRG explants were cultured overnight without NGF and then treated for $1 \mathrm{~h}$ with chariot alone (char) or chariot $+\mathrm{A} 3$ (char $+\mathrm{A} 3$ ) (active ADF/cofilin; $\boldsymbol{A}$ ), or a micropipette coated with chariot $+\mathrm{A} 3$ was brought to one side of a growth cone for 5 min $(\boldsymbol{B})$. Cells were fixed and stained for phospho-ERM (green) and Alexa Fluor 568-phalloidin (red) (phall). The intensities of filopodial phospho-ERM were measured for growth cones $(\boldsymbol{A})$ or the proximal/distal ratio relative to the NGF source (B). $\boldsymbol{C}$, DRGs were transfected with GFP or GFP plus constitutively active LIMK (CA-LIMK), cultured overnight, treated with media or $40 \mathrm{ng} / \mathrm{ml} \mathrm{NGF}$ for 15 min, fixed and stained for phospho-ERM (red), and filopodial intensities were measured. Statistical significance was determined using Student's $t$ test. Data are means \pm SEM; ${ }^{*} p<0.05,{ }^{* *} p<0.01,{ }^{* * *} p<0.001$. Scale bars, $10 \mu \mathrm{m}$.

ERM staining (Fig. 8E). The increase in F-actin following NGF addition was also blocked in growth cones with reduced L1 expression (Fig. $8 \mathrm{~F}$ ). We were prevented from assessing turning to NGF due to the reduced growth cone migration rates in anti-L1 morpholino-expressing neurons after $48 \mathrm{~h}$ (Fig. $8 \mathrm{D}$ ). These data support the idea that $\mathrm{L} 1$ is the major membrane protein that is harnessed by ERM proteins to organize actin filaments and stabilize new actin polymer in filopodia and at the dynamic growth cone leading margin.

The calcium-dependent adhesion receptor $\mathrm{N}$-cadherin is linked to the actin cytoskeleton via catenins (Bard et al., 2008; Shapiro and Weis, 2009). N-cadherin-coated beads on a growth cone surface exhibit retrograde transport (Lambert et al., 2002), and immobilized beads induce local accumulations of F-actin (Bard et al., 2008). To determine whether the effects of L1 knockdown on phospho-ERM and F-actin are specific, we manipulated the calcium concentration of the culture medium to disrupt $\mathrm{N}$-cadherin-dependent growth (Baumgartner et al., 2003; Blackmore and Letourneau, 2006a). DRG explant outgrowth on an $\mathrm{N}$-cadherin substrate is robust in media with $1.0 \mathrm{~mm}$ calcium but significantly reduced in media containing $0.1 \mathrm{~mm}$ calcium We thus grew DRG explants on laminin with 1.0 or $0.1 \mathrm{~mm}$ calcium and measured NGF-induced changes in phospho-ERM and F-actin. As shown in Figure 8G, growth cones cultured with either high or low calcium showed similarly increased filopodial phospho-ERM and phalloidin (F-actin) staining with NGF treatment. These results suggest that the NGF-induced increase in F-actin depends specifically on ERM-mediated linkage of L1 to the cytoskeleton.

\section{Discussion}

ERM (ezrin, moesin, and radixin) proteins are multidomain proteins that link actin filaments to plasma membrane proteins. ERMs also serve as scaffolding for adaptor proteins and signaling molecules. Previous studies of ERM functions in neurons reported that reducing ERM synthesis slowed neurite growth and reduced growth cone size, while inhibiting growth cone ERMs with micro-CALI (chromophore-assisted laser inactivation) locally destabilized lamellipodia (Paglini et al., 1998; Castelo and Jay, 1999). These papers implicated ERM proteins in stabilizing 

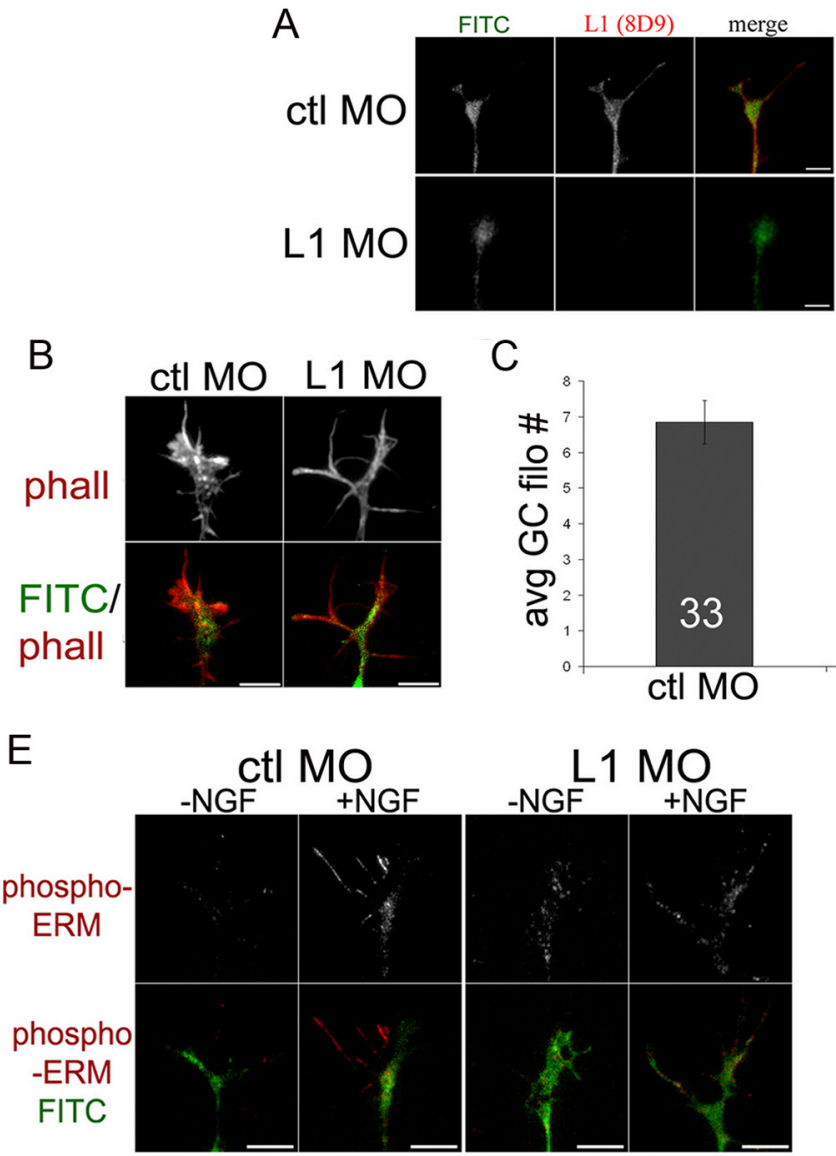

C
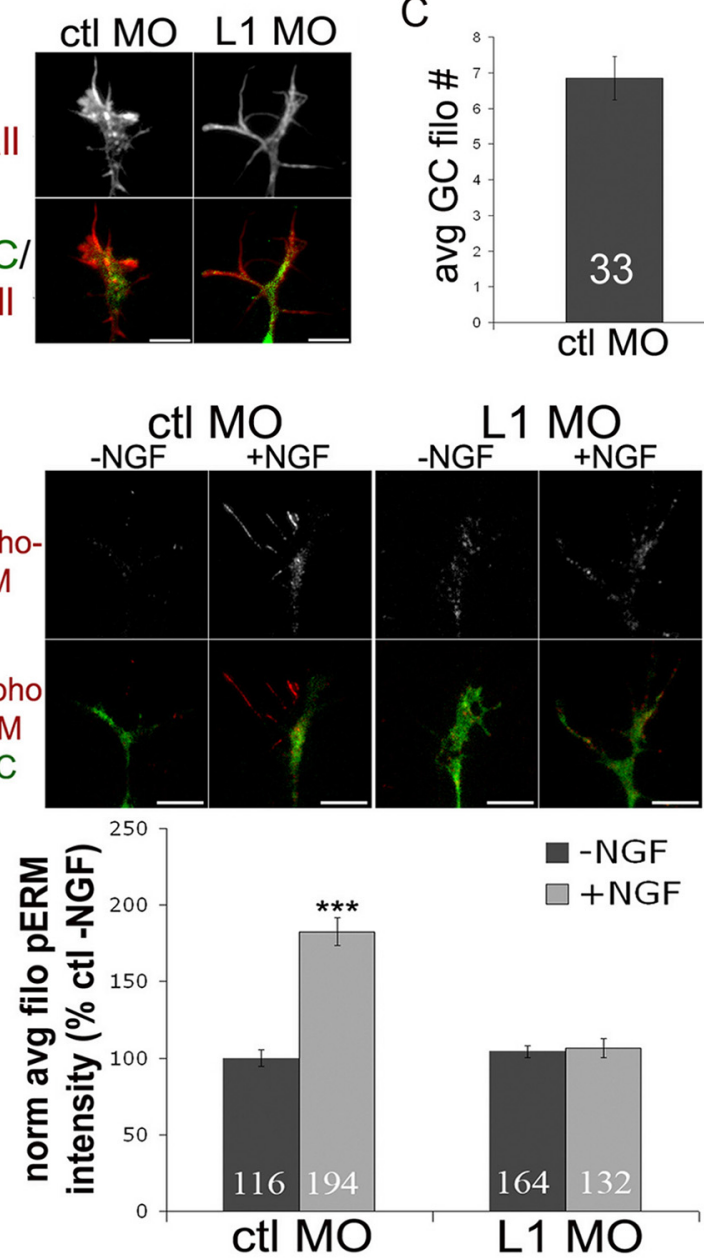

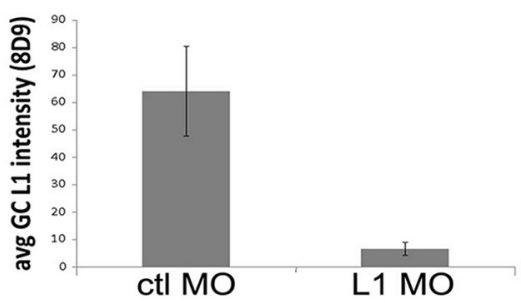

$\mathrm{D}$

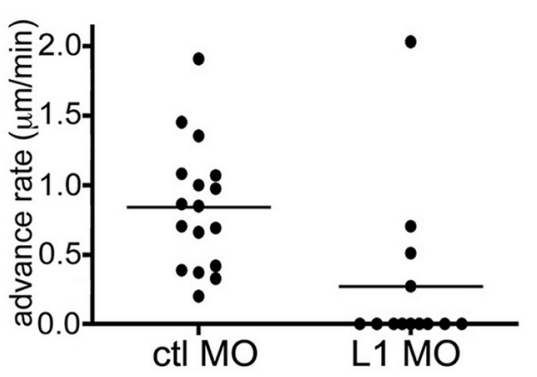

L1 MO

$\mathrm{F}$

L1 MO
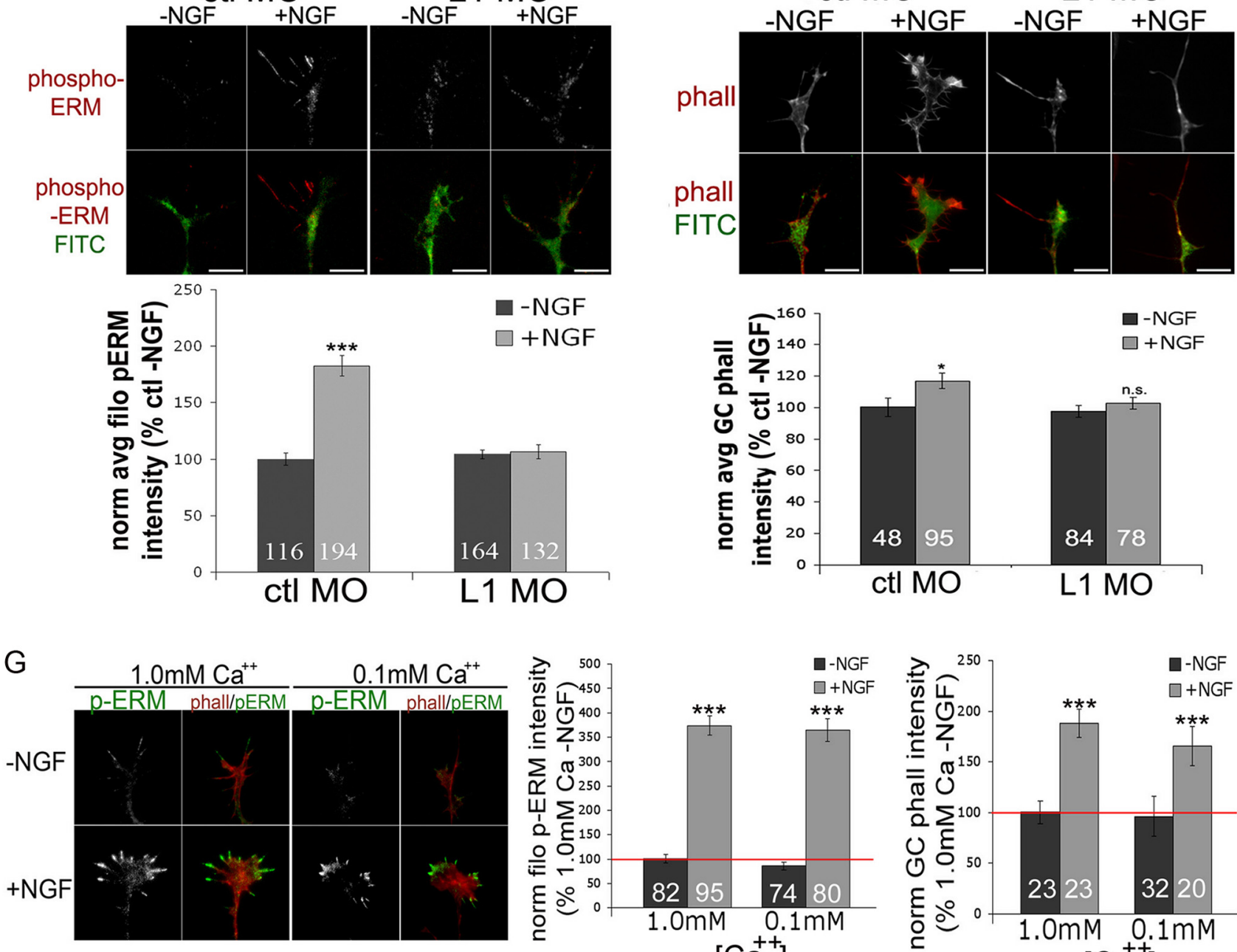

$\left[\mathrm{Ca}^{++}\right]$

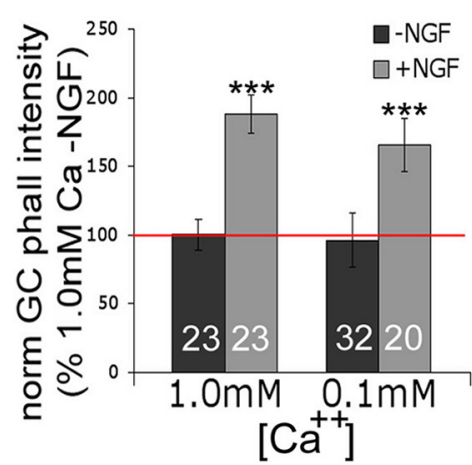

Figure 8. Filopodial phospho-ERM expression is reduced by L1 knockdown. $A-D, D R G$ explants were transfected with FITC-tagged morpholinos against chick L1 (L1 MO) or control (ctl M0) and cultured on laminin for $48 \mathrm{~h}$ with $40 \mathrm{ng} / \mathrm{ml} \mathrm{NGF.} \mathrm{A,} \mathrm{Cells} \mathrm{were} \mathrm{fixed} \mathrm{and} \mathrm{stained} \mathrm{for} \mathrm{L1} \mathrm{(8D9;} \mathrm{red)} \mathrm{and} \mathrm{growth} \mathrm{cone} \mathrm{intensities} \mathrm{were} \mathrm{measured.} \boldsymbol{B}$, Representative growth cones transfected with morpholino and fixed and stained with Alexa Fluor 568-phalloidin. C, Average (avg) number of filopodia (filo) of morpholino-transfected growth cones (GC). D, Growth cone advance rate for control or anti-L1 morpholino. E, $\boldsymbol{F}$, Transfected DRGs were cultured for $24 \mathrm{~h}$ with $40 \mathrm{ng} / \mathrm{mINGF}$ and an additional $24 \mathrm{~h}$ without NGF, and then cells were treated with medium or NGF for 15 min, fixed, stained with phospho-ERM (E) or Alexa Fluor 568 -phalloidin (phall) (F), imaged, and intensities were measured. G, DRG explants were cultured overnight on laminin with medium containing 1.0 or $0.1 \mathrm{~mm}$ calcium. Cells were treated with medium or NGF for 15 min, fixed and stained with Alexa Fluor 568-phalloidin (red) and phospho-ERM (green), and then imaged and intensities were measured. Statistical significance was determined using Student's $t$ test. Data are means \pm SEM; ${ }^{*} p<0.05,{ }^{* * *} p<0.001$. Scale bars, $10 \mu \mathrm{m}$. 
membrane cytoskeletal associations. In other studies, the binding of the cytoplasmic domain of the adhesion molecule L1 to ERM proteins was implicated in mediating neurite branch formation on L1 substrates and in transmitting growth cone traction at L1-mediated adhesion sites (Cheng et al., 2005; Sakurai et al., 2008).

Here we investigated the role of ERMs in chemotactic growth cone guidance. Like previous papers, we found that inhibiting ERM function reduces growth cone migration, filopodia, and actin content. Our new finding is that ERM proteins are necessary for the chemotropic turning of DRG growth cones to multiple attractive guidance cues. Moreover, our results emphasize a key function of ERM proteins in organizing the actin filament network and adhesive properties of the growth cone leading margin.

Activation of ERM proteins by phosphorylation is a critical part of a dynamic response that protrudes a DRG growth cone leading membrane toward an NGF or NT3 source. Within 2 min of neurotrophin stimulation, $\mathrm{P}$-domain phospho-ERM levels are significantly elevated and colocalize with ADF/cofilin and F-actin barbed ends at the growth cone leading margin. Our immunocytochemistry indicates that radixin is the dominant ERM protein in the DRG growth cone leading margin, in agreement with Castelo and Jay (1999). Radixin was initially characterized as an actin barbed-end binding protein (Tsukita et al., 1989), suggesting radixin may link F-actin barbed ends that created by ADF/cofilinmediated filament severing to the leading edge. Thus, the spatially and temporally coordinated activation of $\mathrm{ADF} /$ cofilin and ERM proteins in the growth cone region that is closer to an NGF or NT3 source may connect new actin polymer to the leading edge to effectively harness actin polymerization for driving membrane protrusion toward an attractant source.

We found that trkA mediates the NGF-induced activation of ERM proteins and ADF/cofilin in DRG growth cones. Jeon et al. (2010) found in PC12 cells that NGF-stimulated phosphorylation of moesin is mediated by AKT kinase through its upstream activator PI3 kinase. Similarly, we found that NGF increases AKT phosphorylation in DRG growth cones in a PI3 kinasedependent manner, and the PI3 kinase inhibitor LY294002 reduces the NGF-mediated increase in filopodial phospho-ERM staining. Our data also suggest other trkA-activated kinases, such as PLC and PKC, may also contribute to ERM phosphorylation in response to NGF (Pietromonaco et al., 1998). In addition, our finding that ERM phosphorylation is stimulated by directly increasing ADF/cofilin and F-actin barbed ends in growth cones without NGF-mediated signaling suggests that the active unfolded conformation of ERM proteins is promoted or stabilized by binding to actin polymer. In this, active ERMs could function as positive feedback for continued growth cone protrusion toward an attractive cue source.

The necessity for ERM function in growth cone chemotaxis was indicated by the absence of NGF-induced increase in F-actin content and growth cone turning when ERM function was disrupted by a dominant-negative ERM or siRNA against radixin and moesin. This occurred despite evidence in ERM-inhibited growth cones of trk signaling (increased phospho-AKT) and activation of ADF/cofilin (reduced phospho-ADF/cofilin). However, beyond the disruption of growth cone turning toward NGF, the effects of reduced ERM function indicated a basic role for ERMs in organizing F-actin at the growth cone leading margin.

Along with the reduced total F-actin content in ERMinhibited growth cones, we found a greater fraction of F-actin was located in the growth cone center. In addition, when DN-ERMexpressing growth cones were stimulated with NGF, the active
$\mathrm{ADF} /$ cofilin and resultant F-actin barbed ends were centrally located in growth cones instead of being concentrated at the leading margin. This disrupted actin organization is related to our finding that retrograde flow of F-actin is faster in ERM-inhibited growth cones. This result suggests that F-actin-ERM linkage to the plasma membrane contributes to the molecular "clutch" that counteracts myosin II-powered retrograde actin flow. By linking polymerizing actin filaments to sites of substrate adhesion, the clutch enhances protrusion of the leading margin and transmits traction forces that advance the growth cone. Without ERMmediated linkage of F-actin to the plasma membrane, the clutch may slip more frequently and retrograde F-actin movement from the leading margin may be more rapid than normal, accounting for the more central F-actin localization. A clutch that slips more will also be less effective in exerting traction to advance the growth cone, which is consistent with our observed slower migration of ERM-inhibited growth cones.

In addition to the locally increased actin polymerization, turning of growth cones toward NGF may be promoted by the enhanced expression of adhesion-mediating molecules on filopodia that are closer to an NGF source. After NGF treatment, phospho-ERM and L1 levels are increased and colocalize along filopodial shafts, but when ERM function is disrupted, L1 accumulation along filopodia is blocked. ERM binding may mediate L1 localization to filopodia in several ways. Linkage to ERM proteins may anchor filopodial L1, which reaches filopodia by lateral movement in the membrane and by exocytosis of vesicular L1 (Kamiguchi and Lemmon, 2000; Dequidt et al., 2007). In addition, ERM binding to the RSLE cytoplasmic region of L1 may reduce endocytic removal of L1 from filopodial surfaces by competing with binding of the clathrin-associated adaptor AP-2 (Long et al., 2001; Cheng et al., 2005).

The NGF-induced selective colocalization of L1 and phosphoERM along filopodial shafts illustrates a functional coordination of these two proteins. Homophilic L1 adhesions, as well as heterophilic interactions between L1 and extracellular matrix proteins, mediate neurite outgrowth and neuronal polarization. The concentrated L1/ERM linkage to actin filaments along growth cone filopodia may be particularly effective in providing traction that can turn a growth cone or initiate cytoskeletal rearrangements to form a neuritic branch (Cheng and Lemmon, 2004). Another aspect of the strong filopodial expression of L1 is that L1 may be the major ERM binding partner in dynamic growth cone regions for linking F-actin to the plasma membrane and providing a scaffold for other membrane and signaling proteins. We might not expect that knock-down of L1 synthesis would have blocked the NGF-induced increase in filopodial p-ERM staining if other plasma membrane binding partners for ERMs were available. Furthermore, growth cones of L1-depleted neurons resembled growth cones with disrupted ERM function, as they had few filopodia, a slow migration rate, and a lack of increased F-actin in response to NGF treatment.

NGF also induces local accumulation of $\beta 1$-integrin receptors at filopodial tips distal to the p-ERM that is concentrated along filopodial shafts. However, disrupting ERM function also blocks this response. ERM proteins may act to increase $\beta 1$-integrin expression at filopodial tips by stabilizing parallel bundles of actin filaments that serve as rails for myosin-mediated anterograde transport of $\beta 1$-integrin to filopodial tips (Grabham and Goldberg, 1997; Grabham et al., 2000). Alternatively, ERMs may provide scaffolding for signaling components of integrin adhesions, such as PKA or Epac1 (Gloerich et al., 2010), a guanine nucleotide exchange factor for Rap GTPase, which promotes integrin- 
mediated cell adhesion to ECM molecules (Boettner and Van Aelst, 2009).

In our previous report on the role of $\mathrm{ADF} / \mathrm{cofilin}$ in attractive growth cone turning toward a soluble guidance cue, we showed that the local stimulation of growth cone protrusion involves increased actin polymerization at the leading growth cone margin. Here we show that ERM proteins have an equally important role in attractive guidance by NGF and NT3 by stabilizing new actin polymer at the leading edge so that actin polymerization effectively drives the leading edge forward. In addition, ERM proteins may contribute to the clutch mechanism that transmits mechanical energy from actomyosin contractility to exert traction on substrate adhesions. ERM proteins contribute to the activity of the clutch by linking actin filament to the plasma membrane to slow retrograde actin flow and by mediating the dynamic expression of $\mathrm{L} 1$ and $\beta 1$ integrin adhesion molecules on filopodia. These data point to ERM proteins as central to the coordinated remodeling of growth cone adhesions and F-actin downstream of guidance cues.

In summary, axonal navigation involves signaling by extrinsic guidance cues to regulate the cytoskeletal mechanisms of growth cone migration. The diverse actin binding proteins that orchestrate actin filament dynamics and organization are targets of this signaling, and our studies reported here illustrate how spatial and temporal activation of ADF/cofilin and ERM proteins are coordinated to generate attractive growth cone turning responses to multiple guidance cues. Our conclusion is supported by a recent report that ERM proteins are also activated by the chemoattractant netrin-1 (Antoine-Bertrand et al., 2011).

\section{References}

Agius E, Sagot Y, Duprat AM, Cochard P (1996) Antibodies directed against the beta 1-integrin subunit and peptides containing the IKVAV sequence of laminin perturb neurite outgrowth of peripheral neurons on immature spinal cord substrata. Neuroscience 71:773-786.

Allenspach EJ, Cullinan P, Tong J, Tang Q, Tesciuba AG, Cannon JL, Takahashi SM, Morgan R, Burkhardt JK, Sperling AI (2001) ERM-dependent movement of CD43 defines a novel protein complex distal to the immunological synapse. Immunity 15:739-750.

Alto LT, Havton LA, Conner JM, Hollis ER 2nd, Blesch A, Tuszynski MH (2009) Chemotropic guidance facilitates axonal regeneration and synapse formation after spinal cord injury. Nat Neurosci 12:1106-1113.

Amieva MR, Furthmayr H (1995) Subcellular localization of moesin in dynamic filopodia, retraction fibers, and other structures involved in substrate exploration, attachment, and cell-cell contacts. Exp Cell Res 219:180-196.

Antoine-Bertrand J, Ghogh A, Luangrath V, Bedford FK, Lamarche-Vane N (2011) The activation of ezrin-radixin-moesin proteins is regulated by netrin-1 through Src kinase and RhoA/Rho kinase activities and mediates netrin-1-induced axon outgrowth. Mol Biol Cell 22:3734-3746.

Bard L, Boscher C, Lambert M, Mège RM, Choquet D, Thoumine O (2008) A molecular clutch between the actin flow and N-cadherin adhesions drives growth cone migration. J Neurosci 28:5879-5890.

Baumgartner W, Schütz GJ, Wiegand J, Golenhofen N, Drenckhahn D (2003) Cadherin function probed by laser tweezer and single molecule fluorescence in vascular endothelial cells. J Cell Sci 116:1001-1011.

Baumgartner M, Sillman AL, Blackwood EM, Srivastava J, Madson N, Schilling JW, Wright JH, Barber DL (2006) The Nck-interacting kinase phosphorylates ERM proteins for formation of lamellipodium by growth factors. Proc Natl Acad Sci U S A 103:13391-13396.

Bernard O (2007) Lim kinases, regulators of actin dynamics. Int J Biochem Cell Biol 39:1071-1076.

Blackmore M, Letourneau PC (2006a) Changes within maturing neurons limit axonal regeneration in the developing spinal cord. J Neurobiol 66:348-360.

Blackmore M, Letourneau PC (2006b) L1, betal integrin, and cadherins mediate axonal regeneration in the embryonic spinal cord. J Neurobiol 66:1564-1583.
Boettner B, Van Aelst L (2009) Control of cell adhesion dynamics by Rap1 signaling. Curr Opin Cell Biol 21:684-693.

Bretscher A, Edwards K, Fehon RG (2002) ERM proteins and merlin: integrators at the cell cortex. Nat Rev Mol Cell Biol 3:586-599.

Castelo L, Jay DG (1999) Radixin is involved in lamellipodial stability during nerve growth cone motility. Mol Biol Cell 10:1511-1520.

Chan AY, Raft S, Bailly M, Wyckoff JB, Segall JE, Condeelis JS (1998) EGF stimulates an increase in actin nucleation and filament number at the leading edge of the lamellipod in mammary adenocarcinoma cells. J Cell Sci 111:199-211.

Chan CE, Odde DJ (2008) Traction dynamics of filopodia on compliant substrates. Science 322:1687-1691.

Chen Q, Chen TJ, Letourneau PC, Costa Lda F, Schubert D (2005) Modifier of cell adhesion regulates $\mathrm{N}$-cadherin-mediated cell-cell adhesion and neurite outgrowth. J Neurosci 25:281-290.

Cheng L, Lemmon V (2004) Pathological missense mutations of neural cell adhesion molecule L1 affect neurite outgrowth and branching on an L1 substrate. Mol Cell Neurosci 27:522-530.

Cheng L, Itoh K, Lemmon V (2005) L1-mediated branching is regulated by two ezrin-radixin-moesin (ERM)-binding sites, the RSLE region and a novel juxtamembrane ERM-binding region. J Neurosci 25:395-403.

Crowder RJ, Freeman RS (1998) Phosphatidylinositol 3-kinase and Akt protein kinase are necessary and sufficient for the survival of nerve growth factor-dependent sympathetic neurons. J Neurosci 18:2933-2943.

Dequidt C, Danglot L, Alberts P, Galli T, Choquet D, Thoumine O (2007) Fast turnover of $\mathrm{L} 1$ adhesions in neuronal growth cones involving both surface diffusion and exo/endocytosis of L1 molecules. Mol Biol Cell 8:3131-3143.

Dickson TC, Mintz CD, Benson DL, Salton SR (2002) Functional binding interaction identified between the axonal CAM L1 and members of the ERM family. J Cell Biol 157:1105-1112.

Gallo G (2008) Semaphorin 3A inhibits ERM protein phosphorylation in growth cone filopodia through inactivation of PI3K. Dev Neurobiol 68:926-933.

Gallo G, Letourneau PC (2004) Regulation of growth cone actin filaments by guidance cues. J Neurobiol 58:92-102.

Gautreau A, Poullet P, Louvard D, Arpin M (1999) Ezrin, a plasma membrane-microfilament linker, signals cell survival through the phosphatidylinositol 3-kinase/Akt pathway. Proc Natl Acad Sci U SA 96:7300-7305.

Gehler S, Shaw AE, Sarmiere PD, Bamburg JR, Letourneau PC (2004) Brain-derived neurotrophic factor regulation of retinal growth cone filopodial dynamics is mediated through actin depolymerizing factor/ cofilin. J Neurosci 24:10741-10749.

Genç B, Ozdinler PH, Mendoza AE, Erzurumlu RS (2004) A chemoattractant role for NT-3 in proprioceptive axon guidance. PLoS Biol 2:e403.

Geraldo S, Gordon-Weeks PR (2009) Cytoskeletal dynamics in growthcone steering. J Cell Sci 122:3595-3604.

Gloerich M, Ponsioen B, Vliem MJ, Zhang Z, Zhao J, Kooistra MR, Price LS, Ritsma L, Zwartkruis FJ, Rehmann H, Jalink K, Bos JL (2010) Spatial regulation of cAMP-Epac1 signaling in cell adhesion by ERM proteins. Mol Cell Biol 30:5421-5431.

Gonzalez-Agosti C, Solomon F (1996) Response of radixin to perturbations of growth cone morphology and motility in chick sympathetic neurons in vitro. Cell Motil Cytoskeleton 34:122-136.

Grabham PW, Goldberg DJ (1997) Nerve growth factor stimulates the accumulation of betal integrin at the tips of filopodia in the growth cones of sympathetic neurons. J Neurosci 17:5455-5465.

Grabham PW, Foley M, Umeojiako A, Goldberg DJ (2000) Nerve growth factor stimulates coupling of betal integrin to distinct transport mechanisms in the filopodia of growth cones. J Cell Sci 113:3003-3012.

Hayashi K, Yonemura S, Matsui T, Tsukita S (1999) Immunofluorescence detection of ezrin/radixin/moesin (ERM) proteins with their carboxylterminal threonine phosphorylated in cultured cells and tissues. J Cell Sci 112:1149-1158.

Huber AB, Kolodkin AL, Ginty DD, Cloutier JF (2003) Signaling at the growth cone: ligand-receptor complexes and the control of axon growth and guidance. Annu Rev Neurosci 26:509-563.

Jeon S, Park JK, Bae CD, Park J (2010) NGF-induced moesin phosphorylation is mediated by the PI3K, Racl and Akt and required for neurite formation in PC12 cells. Neurochem Int 56:810-818. 
Kalil K, Dent EW (2005) Touch and go: guidance cues signal to the growth cone cytoskeleton. Curr Opin Neurobiol 15:521-526.

Kamiguchi H, Lemmon V (2000) Recycling of the cell adhesion molecule L1 in axonal growth cones. J Neurosci 20:3676-3686.

Kiryushko D, Berezin V, Bock E (2004) Regulators of neurite outgrowth: role of cell adhesion molecules. Ann N Y Acad Sci 1014:140-154.

Kolodkin AL, Tessier-Lavigne M (2011) Mechanisms and molecules of neuronal wiring: a primer. Cold Spring Harb Perspect Biol 3:a001727.

Lambert M, Choquet D, Mège RM (2002) Dynamics of ligand-induced, Racl-dependent anchoring of cadherins to the actin cytoskeleton. J Cell Biol 157:469-479.

Letourneau PC, Shattuck TA (1989) Distribution and possible interactions of actin-associated proteins and cell adhesion molecules of nerve growth cones. Development 105:505-519.

Lin CH, Forscher P (1993) Cytoskeletal remodeling during growth conetarget interactions. J Cell Biol 121:1369-1383.

Long KE, Asou H, Snider MD, Lemmon V (2001) The role of endocytosis in regulating L1-mediated adhesion. J Biol Chem 276:1285-1290.

Lowery LA, Van Vactor D (2009) The trip of the tip: understanding the growth cone machinery. Nat Rev Mol Cell Biol 10:332-343.

Marsick BM, Flynn KC, Santiago-Medina M, Bamburg JR, Letourneau PC (2010) Activation of ADF/cofilin mediates attractive growth cone turning toward nerve growth factor and netrin-1. Dev Neurobiol 70:565-588.

Matsui T, Yonemura S, Tsukita S, Tsukita S (1999) Activation of ERM proteins in vivo by Rho involves phosphatidyl-inositol 4-phosphate 5-kinase and not ROCK kinases. Curr Biol 9:1259-1262.

McClatchey AI, Fehon RG (2009) Merlin and the ERM proteins-regulators of receptor distribution and signaling at the cell cortex. Trends Cell Biol 19:198-206.

McKerracher L, Chamoux M, Arregui CO (1996) Role of laminin and integrin interactions in growth cone guidance. Mol Neurobiol 12:95-116.

Ming GL, Song HJ, Berninger B, Holt CE, Tessier-Lavigne M, Poo MM (1997) cAMP-dependent growth cone guidance by netrin-1. Neuron 19:1225-1235.

Mintz CD, Dickson TC, Gripp ML, Salton SR, Benson DL (2003) ERMs colocalize transiently with L1 during neocortical axon outgrowth. J Comp Neurol 464:438-448.

Mintz CD, Carcea I, McNickle DG, Dickson TC, Ge Y, Salton SR, Benson DL (2008) ERM proteins regulate growth cone responses to Sema3A. J Comp Neurol 510:351-366.

Morris MC, Depollier J, Mery J, Heitz F, Divita G (2001) A peptide carrier for the delivery of biologically active proteins into mammalian cells. Nat Biotechnol 19:1173-1176.

Neugebauer KM, Tomaselli KJ, Lilien J, Reichardt LF (1988) N-cadherin, NCAM, and integrins promote retinal neurite outgrowth on astrocytes in vitro. J Cell Biol 107:1177-1187.

Niggli V, Rossy J (2008) Ezrin/radixin/moesin: versatile controllers of signaling molecules and of the cortical cytoskeleton. Int J Biochem Cell Biol 40:344-349.
O'Connor R, Tessier-Lavigne M (1999) Identification of maxillary factor, a maxillary process-derived chemoattractant for developing trigeminal sensory axons. Neuron 24:165-178.

Paglini G, Kunda P, Quiroga S, Kosik K, Cáceres A (1998) Suppression of radixin and moesin alters growth cone morphology, motility, and process formation in primary cultured neurons. J Cell Biol 143:443-455.

Pak CW, Flynn KC, Bamburg JR (2008) Actin-binding proteins take the reins in growth cones. Nat Rev Neurosci 9:136-147.

Pietromonaco SF, Simons PC, Altman A, Elias L (1998) Protein kinase C-theta phosphorylation of moesin in the actin-binding sequence. J Biol Chem 273:7594-7603.

Ramer MS, Priestley JV, McMahon SB (2000) Functional regeneration of sensory axons into the adult spinal cord. Nature 403:312-316.

Ramer MS, Bishop T, Dockery P, Mobarak MS, O’Leary D, Fraher JP, Priestley JV, McMahon SB (2002) Neurotrophin-3-mediated regeneration and recovery of proprioception following dorsal rhizotomy. Mol Cell Neurosci 19:239-249.

Roche FK, Marsick BM, Letourneau PC (2009) Protein synthesis in distal axons is not required for growth cone responses to guidance cues. J Neurosci 29:638-652.

Sakurai T, Gil OD, Whittard JD, Gazdoiu M, Joseph T, Wu J, Waksman A, Benson DL, Salton SR, Felsenfeld DP (2008) Interactions between the L1 cell adhesion molecule and ezrin support traction-force generation and can be regulated by tyrosine phosphorylation. J Neurosci Res 86:2602-2614.

Shapiro L, Weis WI (2009) Structure and biochemistry of cadherins and catenins. Cold Spring Harb Perspect Biol 1:a003053.

Simons PC, Pietromonaco SF, Reczek D, Bretscher A, Elias L (1998) C-terminal threonine phosphorylation activates ERM proteins to link the cell's cortical lipid bilayer to the cytoskeleton. Biochem Biophys Res Commun 253:561-565.

Tang P, Cao C, Xu M, Zhang L (2007) Cytoskeletal protein radixin activates integrin alpha(M)beta(2) by binding to its cytoplasmic tail. FEBS Lett 581:1103-1108.

Tsukita S, Yonemura S (1999) Cortical actin organization: lessons from ERM (ezrin/radixin/moesin) proteins. J Biol Chem 274:34507-34510.

Tsukita S, Hieda Y, Tsukita S (1989) A new 82-kD barbed end-capping protein (radixin) localized in the cell-to-cell adherens junction: purification and characterization. J Cell Biol 108:2369-2382.

Tsukita S, Yonemura S, Tsukita S (1997) ERM proteins: head-to-tail regulation of actin-plasma membrane interaction. Trends Biochem Sci 22:53-58

Yang N, Higuchi O, Ohashi K, Nagata K, Wada A, Kangawa K, Nishida E, Mizuno K (1998) Cofilin phosphorylation by LIM-kinase 1 and its role in Rac-mediated actin reorganization. Nature 393:809-812.

Yonemura S, Matsui T, Tsukita S, Tsukita S (2002) Rho-dependent and independent activation mechanisms of ezrin/radixin/moesin proteins: an essential role for polyphosphoinositides in vivo. J Cell Sci 115: $2569-2580$ 